Pacific

Journal of

Mathematics

DILATION OF MARKOVIAN COCYCLES ON A VON NEUMANN ALGEBRA

Debashish Goswami, J. Martin Lindsay, Kalyan B. Sinha, AND Stephen J. WiLls 


\title{
DILATION OF MARKOVIAN COCYCLES ON A VON NEUMANN ALGEBRA
}

\author{
Debashish Goswami, J. Martin Lindsay, Kalyan B. Sinha, \\ AND STEPHEN J. WILLS
}

We consider normal Markovian cocycles on a von Neumann algebra which are adapted to a Fock filtration. Every such cocycle $k$ which is Markov-regular and consists of completely positive contractions is realised as a conditioned *-homomorphic cocycle. This amounts to a stochastic generalisation of a recent dilation result for norm-continuous normal completely positive contraction semigroups. To achieve this stochastic dilation we use the fact that $k$ is governed by a quantum stochastic differential equation whose coefficient matrix has a specific structure, and extend a technique for obtaining stochastic flow generators from Markov semigroup generators, to the context of cocycles. Number/exchange-free dilatability is seen to be related to locality in the case where the cocycle is a Markovian semigroup. In the same spirit unitary dilations of Markov-regular contraction cocycles on a Hilbert space are also described. The paper ends with a discussion of connections with measure-valued diffusion.

\section{Introduction.}

Let $\left(\mathcal{P}_{t}\right)_{t \geq 0}$ be an ultraweakly continuous completely positive contraction semigroup on a von Neumann algebra $\mathcal{M}$. A natural problem is to seek a dilation of $\mathcal{P}$. This has various interpretations (see e.g., [EvL], [Vin], [Küm], [Sa1], [Bha]); the following is a minimum: A family $\left(j_{t}\right)_{t \geq 0}$ of normal *-homomorphisms of $\mathcal{M}$ into a larger algebra $\mathcal{N}$, equipped with a conditional expectation $\mathbb{E}: \mathcal{N} \rightarrow \mathcal{M}$, such that $\mathbb{E} \circ j_{t}=\mathcal{P}_{t}$. One usually further requires $j$ to satisfy a semigroup law. From a physical point of view it is in many situations appropriate to choose the algebra $\mathcal{N}$, to be of the form $\mathcal{M} \otimes \mathcal{B}_{\mathrm{k}}$ where $\mathcal{B}_{\mathrm{k}}=\mathcal{B}\left(\Gamma_{\mathrm{k}}\right)$ and $\Gamma_{\mathrm{k}}$ is the symmetric Fock space over $L^{2}\left(\mathbb{R}_{+} ; \mathrm{k}\right)$ for some Hilbert space $\mathrm{k}$ (see e.g., [AAFL]). In this case the dilation is of Evans-Hudson type ([EH1]) if $\mathbb{E}$ is chosen to be the vacuum expectation and if $j$ satisfies a quantum stochastic (QS) differential equation of the form

$$
d j_{t}=j_{t} \circ \phi_{\beta}^{\alpha} d \Lambda_{\alpha}^{\beta}(t), \quad j_{0}(a)=a \otimes 1,
$$


in which $\Lambda$ denotes the matrix of fundamental QS integrators ([HuP $],[\mathbf{P a r}]$, $[\mathrm{Mey}])$ and $\phi$ is the coefficient matrix of a bounded sesquilinear map $\widehat{\mathrm{k}} \times \hat{\mathrm{k}} \rightarrow$ $\mathcal{B}(\mathcal{A})$, both defined with respect to some basis of the noise dimension space k. This kind of stochastic dilation satisfies a cocycle relation (with respect to the shift of the quantum noise) rather than a semigroup law, however combining $j$ with the shift does result in a semigroup on $\mathcal{M} \otimes \mathcal{B}_{\mathrm{k}}$. In the recent paper [GS] it is shown that an E-H dilation exists when the semigroup $\mathcal{P}$ has bounded generator. A coordinate-free formulation of QS calculus is given in [GS], using Hilbert $W^{*}$-modules, and this copes well with the fact that the noise dimension space $\mathrm{k}$ required for the dilation may in general be nonseparable.

This dilation picture has the following natural generalisation: Let $\mathcal{B}_{J}^{0}=$ $\mathcal{B}\left(\Gamma_{J, \mathrm{k}_{0}}\right)$ where $\Gamma_{J, \mathrm{k}_{0}}$ is the symmetric Fock space over $L^{2}\left(J ; \mathrm{k}_{0}\right)$ for a subinterval $J$ of $\mathbb{R}_{+}$and a Hilbert space $\mathrm{k}_{0}$, writing simply $\mathcal{B}^{0}$ when $J=\mathbb{R}_{+}$. Then $\mathcal{B}^{0}$ carries the filtration of subalgebras $\left(\mathcal{B}_{[0, t}^{0}\right)_{t \geq 0}$ and the semigroup of shifts $\sigma=\left(\sigma_{t}: \mathcal{B}^{0} \rightarrow \mathcal{B}_{[t, \infty[}^{0} \subset \mathcal{B}^{0}\right)_{t \geq 0}$. Suppose that $k=\left(k_{t}: \mathcal{M} \rightarrow \mathcal{M} \otimes \mathcal{B}^{0}\right)_{t \geq 0}$ is an ultraweakly continuous family of completely positive (CP) normal contractions which is adapted to the filtration and is a cocycle with respect to the semigroup of shifts:

$$
k_{s+t}=\widehat{k}_{s} \circ \sigma_{s} \circ k_{t}, \quad k_{0}(a)=a \otimes 1,
$$

where $\widehat{k}_{s}$ is the normal extension of $k_{s}$ to a map $\mathcal{M} \otimes \mathcal{B}_{[s, \infty[}^{0} \rightarrow \mathcal{M} \otimes \mathcal{B}^{0}$ ([LW2]). Is there a Hilbert space $\mathrm{k} \supset \mathrm{k}_{0}$ and a ${ }^{*}$-homomorphic cocycle $j=\left(j_{t}: \mathcal{M} \rightarrow \mathcal{M} \otimes \mathcal{B}_{\mathrm{k}}\right)_{t \geq 0}$ satisfying a QS differential equation of the form (0.1), such that $\mathbb{E}_{0} \circ j_{t}=k_{t}$ where $\mathbb{E}_{0}$ is now the conditional expectation which averages out the quantum noise provided by the supplementary Hilbert space $k \ominus \mathrm{k}_{0}$ ? In other words, subject to regularity, can every CP contraction cocycle be realised as a conditioned ${ }^{*}$-homomorphic cocycle? Note that averaging out all of the noise from such a Markovian cocycle yields an ultraweakly continuous $\mathrm{CP}$ contraction semigroup $\left(\mathcal{P}_{t}=\mathbb{E} \circ k_{t}\right)_{t \geq 0}$, called the Markov semigroup of the cocycle.

The unity of these ideas is further brought out by the recent paper [LW2] where, following [Bra], every such cocycle $k$ which is Markov-regular (that is, whose Markov semigroup $\mathcal{P}$ is norm-continuous) is seen to satisfy a QS differential equation of the form (0.1). In the deterministic case the cocycle reduces to a semigroup and the equation is $d \mathcal{P}_{t}=\mathcal{P}_{t} \circ \theta_{0}^{0} d t$, simply expressing the fact that the semigroup has bounded generator $\theta_{0}^{0}$. We are therefore addressing a stochastic generalisation of the dilation problem for $\mathrm{CP}$ contraction semigroups.

In the present paper we give an affirmative answer to the dilation problem for normal Markov-regular CP contraction cocycles on a von Neumann algebra which are adapted to the Fock filtration, thus extending the results 
of [GS]. The construction is based on the infinitesimal structure of CP flows obtained in $[\mathbf{L i P}]$ and $[\mathbf{L W} \mathbf{1}]$, combined with the techniques introduced in [GS] to obtain a stochastic flow generator from the generator of a CP contraction semigroup. We also consider the dilation problem for Markovian contraction cocycles on a Hilbert space. Again every such cocycle that is Markov-regular is governed by a QS differential equation, this time of the Hudson-Parthasarathy type:

$$
d X_{t}=X_{t} F_{\beta}^{\alpha} d \Lambda_{\alpha}^{\beta}(t), \quad X_{0}=1,
$$

for a matrix $F$ of bounded operators on the Hilbert space ([LW2], $[\mathbf{H u L}])$; moreover the structure of the matrix is once again completely characterised ([LW1], [Fag], [Mo2]).

The plan of the paper is as follows: In Section 1 the basic terminology of quantum stochastic calculus, flows and cocycles is reviewed, and some key results are recalled. Section 2 reviews a characterisation of nonnegative $(2 \times 2)$ operator matrices that is used in the construction of the dilations, and in Section 3 the structure theorems for CP, and CP contraction, flow generators are refined to a form which facilitates stochastic dilation. The *-homomorphic dilations of CP contraction cocycles on a von Neumann algebra, and the unitary dilations of contraction cocycles on a Hilbert space, are constructed in Sections 4 and 6 respectively. In Section 5 CP contraction cocycles which have ${ }^{*}$-homomorphic stochastic dilations involving no number/exchange processes are characterised, and the connection with locality for quantum dynamical semigroups is discussed. The final section contains a discussion of how these ideas might be applied to the theory of measure-valued diffusions.

Dilation for CP flows in a rather different sense arise in the work of Belavkin ([Be2 $])$. There the question raised is one of implementing a CP flow $k$ by conjugation with a solution of a Hudson-Parthasarathy equation of the form (0.2): $k_{t}(a)=X_{t}(a \otimes 1) X_{t}^{*}$ - the flow being on the full algebra of operators on a Hilbert space. In this connection the implementation of CP flows on a von Neumann algebra, by inner perturbations of a ${ }^{*}$-homomorphic flow (in the spirit of $[\mathbf{E H 2}]$ ), is treated elsewhere ([GLW]).

\section{Notation, terminology and background results.}

The symmetric Fock space $\Gamma(\mathrm{H})$ over a Hilbert space $\mathrm{H}$ enjoys the exponential property $\Gamma\left(\mathrm{H}_{1} \oplus \mathrm{H}_{2}\right)=\Gamma\left(\mathrm{H}_{1}\right) \otimes \Gamma\left(\mathrm{H}_{2}\right)$, where the natural isomorphism identifying the two spaces is most economically described in terms of exponential vectors: $\varepsilon\left(f_{1}, f_{2}\right) \longleftrightarrow \varepsilon\left(f_{1}\right) \otimes \varepsilon\left(f_{2}\right)$ ([Par $\left.]\right)$. For a Hilbert space $\mathrm{k}$ and subinterval $J$ of $\mathbb{R}_{+}$we denote $\Gamma\left(L^{2}(J ; \mathrm{k})\right)$ by $\Gamma_{J, \mathrm{k}}$, the algebra of all bounded operators on $\Gamma_{J, \mathrm{k}}$ by $\mathcal{B}_{J, \mathrm{k}}$ and the vacuum vector $\varepsilon(0)$ in $\Gamma_{J, \mathrm{k}}$ by $\Omega_{J, \mathrm{k}}$, abbreviating these to $\Gamma_{\mathrm{k}}, \mathcal{B}_{\mathrm{k}}$ and $\Omega_{\mathrm{k}}$ when $J=\mathbb{R}_{+}$. Two instances of the exponential property that will be important for us are when $\mathrm{k}$ is an orthogonal 
sum of Hilbert spaces $k_{0}$ and $k_{1}$ and when $\mathbb{R}_{+}$is a disjoint union of intervals $J_{1}$ and $J_{2}$. Thus $\Gamma_{\mathrm{k}}=\Gamma_{\mathrm{k}_{0}} \otimes \Gamma_{\mathrm{k}_{1}}, \mathcal{B}_{\mathrm{k}}=\mathcal{B}_{\mathrm{k}_{0}} \otimes \mathcal{B}_{\mathrm{k}_{1}}$ and $\mathcal{B}_{\mathrm{k}_{0}}=\mathcal{B}_{\mathrm{k}_{0}} \otimes 1 \subset \mathcal{B}_{\mathrm{k}}$; $\Gamma_{\mathrm{k}}=\Gamma_{J_{1}, \mathrm{k}} \otimes \Gamma_{J_{2}, \mathrm{k}}, \mathcal{B}_{\mathrm{k}}=\mathcal{B}_{J_{1}, \mathrm{k}} \otimes \mathcal{B}_{J_{2}, \mathrm{k}}$ and $\mathcal{B}_{J_{1}, \mathrm{k}}=\mathcal{B}_{J_{1}, \mathrm{k}} \otimes 1 \subset \mathcal{B}_{\mathrm{k}}$ (where 1 denotes the relevant identity operator) are all identifications that will be invoked without comment.

Let $\mathcal{A}$ be a unital $C^{*}$-algebra acting on an initial Hilbert space $\mathfrak{h}$. In [LW1] and [LW2] the noise dimension space $\mathrm{k}$ is assumed to be separable, the quantum stochastic calculus with infinite degrees of freedom developed there being that initiated by Mohari and Sinha ([MoS $]$ ). An orthonormal basis $\left(e_{i}\right)_{i \geq 1}$ of $\mathrm{k}$ is fixed, and the matrix of quantum stochastic integrators $\left[\Lambda_{\beta}^{\alpha}\right]_{\alpha, \beta \geq 0}$ is defined in terms of these vectors. They fall into four distinct classes: $\Lambda_{0}^{0}$ is the time component; $\Lambda_{0}^{i}=A^{i}$, the $i$ th annihilation component $(i \geq 1) ; \Lambda_{j}^{0}=A_{j}^{\dagger}$, the $j$ th creation component $(j \geq 1)$; and $\Lambda_{j}^{i}=N_{j}^{i}$, the $(i, j)$ th number/exchange (also called gauge or preservation) component $(i, j \geq 1)$. The generator of a quantum stochastic flow is then a (possibly infinite) matrix $\theta=\left[\theta_{\beta}^{\alpha}\right]$ with entries in $\mathcal{B}(\mathcal{A})$. We write $\mathrm{M}_{D}(\mathcal{B}(\mathcal{A})$ ), where $D=1+\operatorname{dim} \mathrm{k}$, for the collection of these mapping matrices, and $\widehat{\mathrm{k}}$ for the Hilbert space $\mathbb{C} \oplus \mathbf{k}$. The Hilbert spaces $\mathfrak{h} \otimes \widehat{k}$ and $\oplus_{\gamma \geq 0} \mathfrak{h}$ may be identified by use of the fixed basis (adding $e_{0}=1 \in \mathbb{C}$ to give a basis of $\widehat{k}$ ). If $\theta$ is the stochastic generator of a $\mathrm{CP}$ contraction flow then the $\theta_{\beta}^{\alpha}$ are components, with respect to this basis, of a bounded linear map, also denoted $\theta$, from $\mathcal{A}$ into $\mathcal{A}^{\prime \prime} \otimes \mathcal{B}(\widehat{\mathrm{k}})([\mathbf{L W} \mathbf{1}]$, Theorem 5.2). Whenever we use this identification and introduce components we shall use the Einstein summation convention and sum over repeated indices; greek indices running from 0 , roman indices from 1 .

This global boundedness property of $\mathrm{CP}$ contraction flow generators connects with the approach in [GS] where QS calculus is reformulated in a coordinate free manner using Hilbert $W^{*}$-modules. The modules encountered in [GS] are all of the form $\mathcal{M} \otimes \mathcal{B}\left(\mathrm{k}_{0} ; \mathrm{k}_{1}\right)$, for Hilbert spaces $\mathrm{k}_{0}$ and $\mathrm{k}_{1}$, and von Neumann algebra $\mathcal{M}$. Such a module may be characterised as the set $\left\{T \in \mathcal{B}\left(\mathfrak{h} \otimes \mathrm{k}_{0} ; \mathfrak{h} \otimes \mathrm{k}_{1}\right):\left(a^{\prime} \otimes 1_{1}\right) T=T\left(a^{\prime} \otimes 1_{0}\right) \forall a^{\prime} \in \mathcal{M}^{\prime}\right\}$, and coincides with the closure of $\mathcal{M} \otimes_{\text {alg. }} \mathcal{B}\left(\mathrm{k}_{0} ; \mathrm{k}_{1}\right)$ in the weak, ultraweak, strong and ultrastrong topologies. We denote the topological dual of $k$ by $k^{*}$ and, since $\mathcal{B}(\mathbb{C} ; \mathrm{k})$ is naturally identified with $\mathrm{k}$, we write $\mathcal{M} \otimes \mathrm{k}$ for $\mathcal{M} \otimes \mathcal{B}(\mathbb{C} ; \mathrm{k})$.

Globally bounded mapping matrices on a $C^{*}$-algebra $\mathcal{A}$ will be written in block matrix form:

$$
\theta=\left[\begin{array}{cc}
\tau & \alpha \\
\chi & \nu-\iota
\end{array}\right]
$$

where $\tau \in \mathcal{B}(\mathcal{A}), \alpha: \mathcal{A} \rightarrow \mathcal{A}^{\prime \prime} \otimes \mathrm{k}^{*}, \chi: \mathcal{A} \rightarrow \mathcal{A}^{\prime \prime} \otimes \mathrm{k}$ and $\nu, \iota: \mathcal{A} \rightarrow \mathcal{A}^{\prime \prime} \otimes \mathcal{B}(\mathrm{k})$ are such that $\theta \in \mathrm{M}_{D}(\mathcal{B}(\mathcal{A}))$. Throughout the paper $\iota$ denotes the map $\iota(a)=a \otimes 1_{\mathrm{k}}$ for the relevant $\mathrm{k}$, and $\widehat{\theta}$ denotes the transformed mapping 
matrix

$$
\widehat{\theta}=\left[\begin{array}{ll}
\tau & \alpha \\
\chi & \nu
\end{array}\right] .
$$

For a subspace $\mathrm{k}_{0}$ of the noise dimension space $\mathrm{k}$, the vacuum conditional expectation $\mathbb{E}_{0}: \mathcal{A}^{\prime \prime} \otimes \mathcal{B}_{\mathrm{k}} \rightarrow \mathcal{A}^{\prime \prime} \otimes \mathcal{B}_{\mathrm{k}_{0}}$ is given by $\mathbb{E}_{0}[c]=E^{*} c E$ where $E$ is the isometry $\mathfrak{h} \otimes \Gamma_{\mathrm{k}_{0}} \ni \xi \mapsto \xi \otimes \Omega_{\mathrm{k}_{0}^{\perp}} \in \mathfrak{h} \otimes \Gamma_{\mathrm{k}}$. When $\mathrm{k}_{0}=\{0\}$ it is denoted simply by $\mathbb{E}$.

Processes on a $C^{*}$-algebra $\mathcal{A}$ with separable noise dimension space $\mathrm{k}$ are defined in generality in $[\mathbf{L W 1}$. Here, apart from in Theorem 3.1, we are exclusively concerned with contraction processes, that is pointwise weakly measurable families of contractions $k=\left(k_{t}\right)_{t \geq 0}$ which are adapted to the Fock filtration: $k_{t}: \mathcal{A} \rightarrow \mathcal{A}^{\prime \prime} \otimes \mathcal{B}_{[0, t[, \mathrm{k}} \otimes 1 \subset \mathcal{A}^{\prime \prime} \otimes \mathcal{B}_{\mathrm{k}}$. When $\mathcal{A}$ is a von Neumann algebra, $k$ is called normal if each map $k_{t}$ is normal. Fock-adapted Markovian cocycles $k$ on a $C^{*}$-algebra $\mathcal{A}$ are defined in $[\mathbf{L W 2}]$, following Bradshaw who defined normal *-homomorphic cocycles on a von Neumann algebra by (1.2) below ([Bra $]$ ). They are required to satisfy a Feller property which includes invariance of the algebra under the maps $\mathcal{P}_{t}=\mathbb{E} \circ k_{t}(t \geq 0)$, which comprise the Markov semigroup of $k$. A Markovian CP contraction cocycle is called Markov-regular when its Markov semigroup is norm continuous. For normal CP contraction processes $k$ on a von Neumann algebra $\mathcal{M}$, the cocycle condition reads

$$
k_{s+t}=\widehat{k}_{s} \circ \sigma_{s} \circ k_{t} \quad(s, t \geq 0),
$$

where $\sigma_{s}$ is the right shift $\mathcal{M} \otimes \mathcal{B}_{\mathrm{k}} \rightarrow \mathcal{M} \otimes \mathcal{B}_{[s, \infty[, \mathrm{k}} \subset \mathcal{M} \otimes \mathcal{B}_{\mathrm{k}}$, and $\widehat{k}_{s}$ is the normal extension of the map $\mathcal{M} \otimes_{\text {alg. }} \mathcal{B}_{[s, \infty[, \mathrm{k}} \rightarrow \mathcal{M} \otimes \mathcal{B}_{\mathrm{k}}$ given by $a \otimes b \mapsto k_{s}(a)(1 \otimes b)$. In the case where $\mathcal{A}$ is a von Neumann algebra, $\mathrm{k}$ is one-dimensional and $k$ is normal, unital and ${ }^{*}$-homomorphic, the following result was established by Bradshaw ([Bra $]$ ). It has been extended to a class of such cocycles whose Markov semigroup is only assumed to be ultraweakly continuous in $[\mathbf{A c M}]$.

Theorem 1.1 ([LW2]). Let $k$ be a CP contraction process on a unital $C^{*}$ algebra $\mathcal{A}$, with separable noise dimension space $\mathrm{k}$. Then the following are equivalent:

(i) $k$ is a Markov-regular cocycle.

(ii) $k$ weakly satisfies a $Q S$ differential equation of the form

$$
d k_{t}=k_{t} \circ \theta_{\beta}^{\alpha} d \Lambda_{\alpha}^{\beta}(t), \quad k_{0}(a)=a \otimes 1
$$

for a mapping matrix $\theta \in \mathrm{M}_{D}(\mathcal{B}(\mathcal{A}))$.

In this case $k$ satisfies the equation strongly and $\theta$ defines a (completely) bounded operator $\mathcal{A} \rightarrow \mathcal{B}(\mathfrak{h} \otimes \widehat{\mathrm{k}})$. Moreover, if $\mathcal{A}$ is a von Neumann algebra then $k$ is normal if and only if $\theta$ is. 
In view of this result we use the terminology Markovian cocycle and stochastic flow interchangeably. Let $k$ and $j$ be a pair of contraction processes on $\mathcal{A}$ with noise dimension spaces $\mathrm{k}_{0}$ and $\mathrm{k}$ respectively, where $\mathrm{k}_{0}$ is a subspace of $\mathrm{k}$. We shall call $j$ a stochastic dilation of $k$ if

$$
k_{t}=\mathbb{E}_{0} \circ j_{t} \quad(t \geq 0) .
$$

Thus, in this terminology, if $k$ is a Markovian cocycle with associated Markov semigroup $\mathcal{P}$ then $k$ is a stochastic dilation of $\mathcal{P}$. When $j$ and $k$ are processes that satisfy QS differential equations of the form (1.3) with noise dimension spaces $\mathrm{k}$ and $\mathrm{k}_{0} \subset \mathrm{k}$ respectively, it is easy to determine when $j$ is a stochastic dilation of $k$ by inspecting their generators.

Lemma 1.2. Let $\theta: \mathcal{A} \rightarrow \mathcal{A}^{\prime \prime} \otimes \mathcal{B}\left(\widehat{\mathrm{k}_{0}}\right)$ and $\phi: \mathcal{A} \rightarrow \mathcal{A}^{\prime \prime} \otimes \mathcal{B}(\widehat{\mathrm{k}})$ be bounded mapping matrices that weakly generate contraction flows $k$ and $j$, with noise dimension spaces $\mathrm{k}_{0}$ and $\mathrm{k}=\mathrm{k}_{0} \oplus \mathrm{k}_{1}$ respectively. Then $j$ is a stochastic dilation of $k$ if and only if $\phi$ has block matrix form

$$
\phi=\left[\begin{array}{ll}
\theta & * \\
* & *
\end{array}\right] \text {. }
$$

Proof. If $j$ is a stochastic dilation of $k$ then, for all $u, v \in \mathfrak{h}$ and $f^{0}, g^{0} \in$ $L^{2}\left(\mathbb{R}_{+} ; \mathrm{k}_{0}\right)$,

$$
\left\langle u \varepsilon\left(f^{0}\right), k_{t}(a) v \varepsilon\left(g^{0}\right)\right\rangle=\left\langle u \varepsilon\left(f^{0}, 0\right), j_{t}(a) v \varepsilon\left(g^{0}, 0\right)\right\rangle .
$$

Applying the first fundamental formula of quantum stochastic calculus to each side, differentiating the resulting expressions at $t=0$, and varying the test functions $f^{0}$ and $g^{0}$ reveals that $\phi$ has the form (1.4). Conversely, if $\phi$ has the form (1.4) then the process $\mathbb{E}_{0} \circ j$ is also a weak solution of the QS differential equation satisfied by $k$. Thus by uniqueness of solutions ([LW1], Theorem 3.1) $k=\mathbb{E}_{0} \circ j$.

For a unital $C^{*}$-algebra $\mathcal{A}$ acting on the Hilbert space $\mathfrak{h}$, a representation $(\pi, \mathrm{H})$ of $\mathcal{A}$, and operators $R \in \mathcal{B}(\mathfrak{h} ; \mathrm{H})$ and $H \in \mathcal{B}(\mathfrak{h})$ we write $\delta_{R, \pi}$ and $\mathcal{L}_{R, \pi, H}$ for the operators given by

$$
\begin{gathered}
\delta_{R, \pi}(a)=R a-\pi(a) R, \\
\mathcal{L}_{R, \pi, H}(a)=R^{*} \pi(a) R-\frac{1}{2}\left\{R^{*} R, a\right\}+i[H, a] .
\end{gathered}
$$

Thus $\delta_{R, \pi}: \mathcal{A} \rightarrow \mathcal{B}(\mathfrak{h} ; \mathrm{H})$ is a $\pi$-derivation, and $\mathcal{L}_{R, \pi, H}: \mathcal{A} \rightarrow \mathcal{B}(\mathfrak{h})$ satisfies

$$
\partial \mathcal{L}_{R, \pi, H}(a, b)=\delta_{R, \pi}(a)^{*} \delta_{R, \pi}(b)+a^{*} R^{*} \pi(1)^{\perp} R b
$$

where, for a linear map $\tau: \mathcal{A} \rightarrow \mathcal{B}(\mathfrak{h}), \partial \tau: \mathcal{A} \times \mathcal{A} \rightarrow \mathcal{B}(\mathfrak{h})$ is the sesquilinear map defined by

$$
\partial \tau(a, b)=\tau\left(a^{*} b\right)-a^{*} \tau(b)-\tau\left(a^{*}\right) b+a^{*} \tau(1) b .
$$

The map $\tau$ is called real if it satisfies $\tau^{\dagger}=\tau$, where $\tau^{\dagger}$ is defined by $\tau^{\dagger}(a)=$ $\tau\left(a^{*}\right)^{*}$. The following result in contained in $[\mathbf{C h E}]$ : 
Theorem 1.3. Let $(\tau, \rho, \mathrm{H}, \delta)$ consist of a map $\tau \in \mathcal{B}(\mathcal{A})$, a representation $(\rho, \mathrm{H})$ of $\mathcal{A}$, and a $\rho$-derivation $\delta: \mathcal{A} \rightarrow \mathcal{B}(\mathfrak{h} ; \mathrm{H})$ satisfying $\partial \tau(a, b)=$ $\delta(a)^{*} \delta(b)$ and $\delta(1)=0$. Then there is an operator $R \in \mathcal{B}(\mathfrak{h} ; \mathrm{H})$ which lies in the ultraweak closure of $\operatorname{Lin}\{\delta(a) b: a, b \in \mathcal{A}\}$ and an element $h \in \mathcal{A}^{\prime \prime}$ such that

$$
\delta(\cdot)=\delta_{R, \rho}(\cdot) \text { and } \tau(\cdot)=\mathcal{L}_{R, \rho, h}(\cdot)+\frac{1}{2}\{\tau(1), \cdot\} .
$$

If $\tau$ is real then $h$ may be chosen so that $h=h^{*}$.

\section{Nonnegative operator matrices.}

In this section we recall a characterisation of nonnegative operator block matrices that will be exploited in the construction of dilations. Parts (a) and (b) of the lemma below are classical and can be traced back as far as Schur - see $[\mathbf{F o F}]$, p. 547 for historical comments. We include a proof for the convenience of the reader. Part (c) is the special case required for the following section.

Lemma 2.1. Let $T \in \mathcal{B}\left(\mathrm{H}_{1} \oplus \mathrm{H}_{2}\right)$ for Hilbert spaces $\mathrm{H}_{1}$ and $\mathrm{H}_{2}$.

(a) The following are equivalent:

(i) $T \geq 0$.

(ii) In block matrix form

$$
T=\left[\begin{array}{cc}
A & A^{1 / 2} V D^{1 / 2} \\
D^{1 / 2} V^{*} A^{1 / 2} & D
\end{array}\right]
$$

where $A, D \geq 0$ and $V \in \mathcal{B}\left(\mathrm{H}_{2} ; \mathrm{H}_{1}\right)$ is a contraction.

(b) There is a representation (2.1) in which

$$
\text { Ker } V \supset \operatorname{Ker} D \text { and } \operatorname{Ran} V \subset \overline{\operatorname{Ran}} A \text {; }
$$

this $V$ is unique.

(c) If the Hilbert spaces are of the form $\mathrm{H} \otimes \mathrm{h}_{1}$ and $\mathrm{H} \otimes \mathrm{h}_{2}$ respectively, and $T$ belongs to $\mathcal{C} \otimes \mathcal{B}\left(\mathrm{h}_{1} \oplus \mathrm{h}_{2}\right)$ for a von Neumann algebra $\mathcal{C}$ acting on $\mathrm{H}$, then the unique contraction $V$ satisfying (2.1) and (2.2) belongs to the $W^{*}$-module $\mathcal{C} \otimes \mathcal{B}\left(\mathrm{h}_{2} ; \mathrm{h}_{1}\right)$.

Proof. If (a)(ii) holds then $T$ may be written

$$
\left[\begin{array}{cc}
A^{1 / 2} & 0 \\
0 & D^{1 / 2}
\end{array}\right]\left\{\left[\begin{array}{l}
V \\
1
\end{array}\right]\left[\begin{array}{cc}
V^{*} & 1
\end{array}\right]+\left[\begin{array}{cc}
1-V V^{*} & 0 \\
0 & 0
\end{array}\right]\right\}\left[\begin{array}{cc}
A^{1 / 2} & 0 \\
0 & D^{1 / 2}
\end{array}\right]
$$

which is manifestly nonnegative. 
Conversely, if $T$ is nonnegative with block matrix form $\left[\begin{array}{cc}A & B \\ C & D\end{array}\right]$ then obviously $A, D \geq 0$ and $C=B^{*}$. By the Cauchy-Schwarz inequality,

$$
\begin{aligned}
|\langle\xi, B \eta\rangle|^{2} & =\left|\left\langle\left(\begin{array}{l}
\xi \\
0
\end{array}\right), T\left(\begin{array}{l}
0 \\
\eta
\end{array}\right)\right\rangle\right|^{2} \\
& \leq\left\langle\left(\begin{array}{l}
\xi \\
0
\end{array}\right), T\left(\begin{array}{l}
\xi \\
0
\end{array}\right)\right\rangle\left\langle\left(\begin{array}{l}
0 \\
\eta
\end{array}\right), T\left(\begin{array}{l}
0 \\
\eta
\end{array}\right)\right\rangle=\left\|A^{1 / 2} \xi\right\|^{2}\left\|D^{1 / 2} \eta\right\|^{2} .
\end{aligned}
$$

First note that this implies that

$$
\|B \eta\| \leq\left\|A^{1 / 2}\right\|\left\|D^{1 / 2} \eta\right\| \quad \forall \eta \in \mathrm{H}_{2},
$$

from which it follows that there is a (unique) operator $J \in \mathcal{B}\left(\mathrm{H}_{2} ; \mathrm{H}_{1}\right)$ satisfying

$$
B=J D^{1 / 2} \text { and } \operatorname{Ker} J \supset \operatorname{Ker} D^{1 / 2} .
$$

Now substitute this back into the Cauchy-Schwarz estimate:

$$
\left|\left\langle J^{*} \xi, D^{1 / 2} \eta\right\rangle\right| \leq\left\|A^{1 / 2} \xi\right\|\left\|D^{1 / 2} \eta\right\| .
$$

Since $\overline{\operatorname{Ran}} J^{*}=(\operatorname{Ker} J)^{\perp} \subset\left(\operatorname{Ker} D^{1 / 2}\right)^{\perp}=\overline{\operatorname{Ran}} D^{1 / 2}$, this implies that

$$
\left\|J^{*} \xi\right\| \leq\left\|A^{1 / 2} \xi\right\| \quad \forall \xi \in \mathrm{H}_{1},
$$

from which it follows that there is a (unique) contraction $W \in \mathcal{B}\left(\mathrm{H}_{1} ; \mathrm{H}_{2}\right)$ satisfying

$$
J^{*}=W A^{1 / 2} \text { and } \operatorname{Ker} W \supset \operatorname{Ker} A^{1 / 2} .
$$

Since $W$ also satisfies $\overline{\operatorname{Ran}} W=\overline{\operatorname{Ran}} J^{*}$, if we put $V=W^{*}$ then we have $B=J D^{1 / 2}=A^{1 / 2} V D^{1 / 2}$ and $\operatorname{Ker} V=(\operatorname{Ran} W)^{\perp}=\operatorname{Ker} J \supset \operatorname{Ker} D^{1 / 2}=$ Ker $D$. Also Ran $V \subset(\operatorname{Ker} W)^{\perp} \subset\left(\operatorname{Ker} A^{1 / 2}\right)^{\perp}=\overline{\operatorname{Ran}} A^{1 / 2}=\overline{\operatorname{Ran}} A$, so (a)(ii) follows along with the first part of (b).

The uniqueness of $V$ subject to (2.2) follows from the identity

$$
\left\langle A^{1 / 2} \xi, V D^{1 / 2} \eta\right\rangle=\left\langle\left(\begin{array}{l}
\xi \\
0
\end{array}\right), T\left(\begin{array}{l}
0 \\
\eta
\end{array}\right)\right\rangle .
$$

Under the conditions of (c) obviously $A \in \mathcal{C} \otimes \mathcal{B}\left(\mathrm{h}_{1}\right)$ and $D \in \mathcal{C} \otimes \mathcal{B}\left(\mathrm{h}_{2}\right)$. Moreover

$$
\left\langle\left(c^{\prime} \otimes 1_{1}\right) A^{1 / 2} \xi, V\left(d^{\prime} \otimes 1_{2}\right) D^{1 / 2} \eta\right\rangle=\left\langle\left(c^{\prime} \otimes 1\right)\left(\begin{array}{l}
\xi \\
0
\end{array}\right), T\left(d^{\prime} \otimes 1\right)\left(\begin{array}{l}
0 \\
\eta
\end{array}\right)\right\rangle
$$

for $c^{\prime}, d^{\prime} \in \mathcal{C}^{\prime}$, where $1_{1}, 1_{2}$ and 1 are the identities on $\mathrm{h}_{1}, \mathrm{~h}_{2}$ and $\mathrm{h}_{1} \oplus \mathrm{h}_{2}$ respectively; it follows that $V \in \mathcal{C} \otimes \mathcal{B}\left(\mathrm{h}_{2} ; \mathrm{h}_{1}\right)$. 


\section{Structural form for a flow generator.}

In this section we refine the characterisation of $\mathrm{CP}$ flow generators on a unital $C^{*}$-algebra $\mathcal{A}$ found in $[\mathbf{L i P}]$ and $[\mathbf{L W} \mathbf{1}]$ in such a way that, when $\mathcal{A}$ is a von Neumann algebra and the flow is a normal contraction flow, the constituents of the generator may be used to determine a ${ }^{*}$-homomorphic stochastic dilation. The refinement uses the technique introduced in [GS] for dilating $\mathrm{CP}$ contraction semigroups.

Fix a separable noise dimension space $\mathrm{k}_{0}$, with basis $\left(e_{i}\right)_{i \geq 1}$. To begin with we do not assume that $k$ is a contraction flow. Therefore (when $\mathrm{k}_{0}$ is infinite dimensional), its generator $\theta$ need not be bounded, and we must work with its components $\theta_{\beta}^{\alpha}$. Following $(1.1)^{\prime}$ write $\left[\begin{array}{cc}\tau & \chi_{j}^{\dagger} \\ \chi^{i} & \nu_{j}^{i}\end{array}\right]$ for the $(2 \times 2)$ matrix of maps $\left[\begin{array}{cc}\theta_{0}^{0} & \theta_{j}^{0} \\ \theta_{0}^{i} & \widehat{\theta}_{j}^{i}\end{array}\right], i, j \geq 1$ when $\theta$ is real. Let $\mathfrak{h}_{00}$ be the subspace of $\oplus_{\gamma \geq 0} \mathfrak{h}=\mathfrak{h} \otimes \widehat{k_{0}}$ consisting of vectors with only finitely many nonzero components with respect to the chosen basis.

Theorem 3.1. Let $\theta$ be a mapping matrix on the unital $C^{*}$ - algebra $\mathcal{A}$ that weakly generates a stochastic flow $k$.

(a) The following are equivalent:

(i) $k$ is completely positive.

(ii) $\theta$ is real and there is a quintuple $\mathcal{R}=\left(\mathrm{h}, \pi, h, d,\left\{w_{j}\right\}\right)$ consisting of a Hilbert space $\mathrm{h}, a^{*}$-homomorphism $\pi: \mathcal{A} \rightarrow \mathcal{A}^{\prime \prime} \otimes \mathcal{B}(\mathrm{h})$ and operators $h=h^{*} \in \mathcal{A}^{\prime \prime}, d \in \mathcal{A}^{\prime \prime} \otimes \mathrm{h}$ and $w_{j} \in \mathcal{A}^{\prime \prime} \otimes \mathrm{h}, j \geq 1$, such that

$$
\begin{gathered}
{\left[\begin{array}{cc}
\tau(a) & \chi_{j}^{\dagger}(a) \\
\chi^{i}(a) & \nu_{j}^{i}(a)
\end{array}\right]=\left[\begin{array}{cc}
\mathcal{L}(a)+\frac{1}{2}\{t, a\} & \delta^{\dagger}(a) w_{j}+a\left(c^{j}\right)^{*} \\
w_{i}^{*} \delta(a)+c^{i} a & w_{i}^{*} \pi(a) w_{j}
\end{array}\right]} \\
\pi(1) d=d \text { and } \pi(1) w_{j}=w_{j} \quad \forall j \\
\operatorname{Ran} \pi(1)=\mathrm{H}
\end{gathered}
$$

where $t=\tau(1), c^{i}=\chi^{i}(1), \delta=\delta_{d, \pi}, \mathcal{L}=\mathcal{L}_{d, \pi, h}$ and $\mathrm{H}=\overline{\mathrm{H}_{0}}$ with

$$
\mathrm{H}_{0}=\operatorname{Lin}\left\{\delta(a) u^{0}+\pi(a) w_{j} u^{j}: a \in \mathcal{A},\left(u^{\alpha}\right) \in \mathfrak{h}_{00}\right\} .
$$

(b) If $\mathcal{R}_{1}$ and $\mathcal{R}_{2}$ are quintuples satisfying (3.1) then there is a unique partial isometry $V: \mathfrak{h} \otimes \mathrm{h}_{1} \rightarrow \mathfrak{h} \otimes \mathrm{h}_{2}$ satisfying

$$
\begin{gathered}
V^{*} V=\pi_{1}(1) ; \quad V V^{*}=\pi_{2}(1) \\
\pi_{2}(a)=V \pi_{1}(a) V^{*} ; \quad \delta_{2}=V \delta_{1} ; \quad w_{j}^{2}=V w_{j}^{1} .
\end{gathered}
$$

Moreover $V \in \mathcal{A}^{\prime \prime} \otimes \mathcal{B}\left(\mathrm{h}_{1} ; \mathrm{h}_{2}\right)$.

(c) If $\mathcal{A}$ is a von Neumann algebra and $\mathcal{R}$ is a quintuple satisfying (3.1), then the representation $(\pi, \mathfrak{h} \otimes \mathrm{h})$ is normal if and only if each $\theta_{\beta}^{\alpha}$ is. 
Proof. The implication (a)(ii) $\Rightarrow($ a)(i) is contained in [LW1], Theorem 4.1.

(a)(i) $\Rightarrow$ (a)(ii): Suppose that $k$ is completely positive. Then, by Theorem 4.1 of $[\mathbf{L W} \mathbf{1}], \theta$ is real and there is a quadruple $\mathcal{Q}=\left(\rho, \mathcal{H}, \gamma,\left\{W_{i}\right\}\right)$ consisting of a representation $(\rho, \mathcal{H})$ of $\mathcal{A}$, a $\rho$-derivation $\gamma: \mathcal{A} \rightarrow \mathcal{B}(\mathfrak{h} ; \mathcal{H})$ and a family of operators $\left\{W_{i}: i \geq 1\right\}$ in $\mathcal{B}(\mathfrak{h} ; \mathcal{H})$ such that

$$
\left[\begin{array}{cc}
\partial \tau(a, b) & \chi_{j}^{\dagger}(a) \\
\chi^{i}(a) & \nu_{j}^{i}(a)
\end{array}\right]=\left[\begin{array}{cc}
\gamma(a)^{*} \gamma(b) & \gamma^{\dagger}(a) W_{j}+a \chi_{j}^{\dagger}(1) \\
W_{i}^{*} \gamma(a)+\chi^{i}(1) a & W_{i}^{*} \rho(a) W_{j}
\end{array}\right]
$$
$\rho$ is unital

$$
\mathcal{H}=\overline{\mathcal{H}_{0}}
$$

where $\mathcal{H}_{0}=\operatorname{Lin}\left\{\gamma(a) u^{0}+\rho(a) W_{i} u^{i}: a \in \mathcal{A},\left(u^{\alpha}\right) \in \mathfrak{h}_{00}\right\}$. For each unitary $u^{\prime} \in \mathcal{A}^{\prime}$ define bounded linear operators $\gamma^{u^{\prime}}: \mathcal{A} \rightarrow \mathcal{B}(\mathfrak{h} ; \mathcal{H})$ and $W_{j}^{u^{\prime}} \in \mathcal{B}(\mathfrak{h} ; \mathcal{H})$ by $\gamma^{u^{\prime}}(a)=\gamma(a) u^{\prime}$ and $W_{j}^{u^{\prime}}=W_{j} u^{\prime}$, and note the following relations:

$$
\begin{gathered}
\gamma^{u^{\prime}}(a)^{*} \gamma^{u^{\prime}}(b)=u^{\prime *} \partial \tau(a, b) u^{\prime}=\partial \tau(a, b)=\gamma(a)^{*} \gamma(b) ; \\
\left(W_{i}^{u^{\prime}}\right)^{*} \gamma^{u^{\prime}}(a)=u^{\prime *} W_{i}^{*} \gamma(a) u^{\prime}=u^{\prime *}\left(\chi^{i}(a)-\chi^{i}(1) a\right) u^{\prime}=W_{i}^{*} \gamma(a) ; \\
\left(W_{i}^{u^{\prime}}\right)^{*} \rho(a) W_{j}^{u^{\prime}}=u^{\prime *} W_{i}^{*} \rho(a) W_{j} u^{\prime}=u^{\prime *} \nu_{j}^{i}(a) u^{\prime}=\nu_{j}^{i}(a) ; \\
\mathcal{H}_{0}^{u^{\prime}}=\mathcal{H}_{0} .
\end{gathered}
$$

In other words the quadruple $\mathcal{Q}^{u^{\prime}}=\left(\rho, \mathcal{H}, \gamma^{u^{\prime}},\left\{W_{i}^{u^{\prime}}\right\}\right)$ also satisfies (3.3). Hence, by the uniqueness part of Theorem 4.1 in [LW1], there is a unique unitary operator $\rho^{\prime}\left(u^{\prime}\right)$ on $\mathcal{H}$ such that

$$
\begin{gathered}
\rho^{\prime}\left(u^{\prime}\right) W_{i}=W_{i} u^{\prime} ; \quad \rho^{\prime}\left(u^{\prime}\right) \gamma(a)=\gamma(a) u^{\prime} ; \\
\rho^{\prime}\left(u^{\prime}\right) \rho(a)=\rho(a) \rho^{\prime}\left(u^{\prime}\right) .
\end{gathered}
$$

The resulting map $\rho^{\prime}$ is easily seen to be a unitary representation of the group of unitaries in $\mathcal{A}^{\prime}$ by checking matrix elements against vectors from the dense subspace $\mathcal{H}_{0}$. It follows that $\rho^{\prime}$ extends linearly to a normal, unital representation of $\mathcal{A}^{\prime}$. Hence ([Dix], p. 61) there is a Hilbert space $\mathrm{h}$ and an isometry $V: \mathcal{H} \rightarrow \mathfrak{h} \otimes \mathrm{h}$ such that $\rho^{\prime}\left(x^{\prime}\right)=V^{*}\left(x^{\prime} \otimes 1\right) V$ and $p:=V V^{*} \in \mathcal{A}^{\prime \prime} \otimes \mathcal{B}(\mathrm{h})$. Put $\mathrm{H}=V \mathcal{H}$ and define $\pi^{\prime}: \mathcal{A}^{\prime} \rightarrow \mathcal{B}(\mathfrak{h} \otimes \mathrm{h}), \pi: \mathcal{A} \rightarrow$ $\mathcal{B}(\mathfrak{h} \otimes \mathrm{h}), \delta: \mathcal{A} \rightarrow \mathcal{B}(\mathfrak{h} ; \mathfrak{h} \otimes \mathrm{h})$, and $w_{j} \in \mathcal{B}(\mathfrak{h} ; \mathfrak{h} \otimes \mathbf{h})$ for $j \geq 1$, by

$$
\pi^{\prime}\left(x^{\prime}\right)=\left(x^{\prime} \otimes 1\right) p, \pi(a)=V \rho(a) V^{*}, \delta(a)=V \gamma(a), w_{j}=V W_{j} .
$$


Since $p \in \mathcal{A}^{\prime \prime} \otimes \mathcal{B}(\mathrm{h})$, algebraic manipulations applied to (3.4) reveal the following identities:

$$
\begin{gathered}
\pi(1)=p ; \quad p \delta(a)=\delta(a) ; \quad p w_{j}=w_{j}, \\
\pi(a)\left(x^{\prime} \otimes 1\right)=\left(x^{\prime} \otimes 1\right) \pi(a)=\pi^{\prime}\left(x^{\prime}\right) \pi(a), \\
\delta(a) x^{\prime}=\left(x^{\prime} \otimes 1\right) \delta(a), \\
w_{j} x^{\prime}=\left(x^{\prime} \otimes 1\right) w_{j} .
\end{gathered}
$$

Thus $\pi(\mathcal{A}) \subset \mathcal{A}^{\prime \prime} \otimes \mathcal{B}(\mathrm{h}), \delta(\mathcal{A}) \subset \mathcal{A}^{\prime \prime} \otimes \mathrm{h}$ and $w_{j} \in \mathcal{A}^{\prime \prime} \otimes \mathrm{h}$. Moreover $\pi$ is a representation, $\delta$ a $\pi$-derivation, and

$$
\operatorname{Ran} \pi(1)=\operatorname{Ran} V=\overline{V\left(\mathcal{H}_{0}\right)}=\overline{\mathrm{H}_{0}}=\mathrm{H} .
$$

Now $\delta(1)=0$ and $\delta(a)^{*} \delta(b)=\partial \tau(a, b)$, from its definition. Thus by Theorem 1.3 there is some $d \in \overline{\operatorname{Lin}}^{\mathrm{ww}}\{\delta(a) b: a, b\} \subset \mathcal{A}^{\prime \prime} \otimes \mathrm{h}$ and $h=h^{*} \in \mathcal{A}^{\prime \prime}$ such that $\delta=\delta_{d, \pi}$ and $\tau(\cdot)=\mathcal{L}_{d, \pi, h}(\cdot)+\frac{1}{2}\{\tau(1), \cdot\}$. Note also that $\pi(1) d=d$ since $\delta(1)=0$, which completes part (a).

(b) Writing $\mathrm{H}_{i}$ for $\operatorname{Ran} \pi_{i}(1) \subset \mathfrak{h} \otimes \mathrm{h}_{i}, i=1,2$, Theorem 4.1 of [LW1] ensures the existence of a unique unitary operator $V_{0}: \mathrm{H}_{1} \rightarrow \mathrm{H}_{2}$ satisfying

$$
V_{0} w_{j}^{1}=w_{j}^{2} ; \quad V_{0} \delta_{1}=\delta_{2} ; \quad V_{0} \pi_{1}(a)=\pi_{2}(a) V_{0} .
$$

Let $V$ be the unique extension of $V_{0}$ to an operator $V: \mathfrak{h} \otimes \mathrm{h}_{1} \rightarrow \mathfrak{h} \otimes \mathrm{h}_{2}$ that satisfies (3.2i). Then $V$ satisfies (3.2ii), and is thus clearly the unique partial isometry satisfying (3.2). That $V$ belongs to $\mathcal{A}^{\prime \prime} \otimes \mathcal{B}\left(\mathrm{h}_{1} ; \mathrm{h}_{2}\right)$ follows from the identity

$$
\left(a^{\prime} \otimes 1_{i}\right)\left(\delta_{i}(a) u^{0}+\pi_{i}(a) w_{j}^{i} u^{j}\right)=\delta_{i}(a) a^{\prime} u^{0}+\pi_{i}(a) w_{j}^{i} a^{\prime} u^{j} .
$$

(c) In one direction this is trivial. To obtain normality of $\pi$ from the normality of all of the $\theta_{\beta}^{\alpha}$ it is enough to consider the restriction of $\pi$ to $\pi(1)(\mathfrak{h} \otimes \mathrm{h})$ and apply [LW1], Theorem 4.1(d).

Remark. If $\mathcal{A}$ is a von Neumann algebra, the initial space $\mathfrak{h}$ is separable, and each $\theta_{\beta}^{\alpha}$ is ultraweakly continuous, then Proposition 4.2 of [LW1] implies that the representation space $\mathcal{H}$ in the quadruple $\mathcal{Q}$ is separable too, so that in particular the von Neumann algebra $\rho^{\prime}\left(\mathcal{A}^{\prime}\right)^{\prime}$ is $\sigma$-finite. In this case we may assume that the Hilbert space $\mathrm{h}$ in Theorem 3.1 is separable too (see [Dix], p. 62).

Theorem 3.2. Let $\theta$ be a mapping matrix on the unital $C^{*}$-algebra $\mathcal{A}$ that weakly generates a stochastic flow $k$. The following are equivalent:

(i) $k$ is a completely positive contraction cocycle.

(ii) $\theta$ is real and bounded, and there is a sextuple $\mathcal{S}=(\mathrm{h}, \pi, h, d, w, v)$ consisting of a Hilbert space $\mathrm{h}, a^{*}$-homomorphism $\pi: \mathcal{A} \rightarrow \mathcal{A}^{\prime \prime} \otimes \mathcal{B}(\mathrm{h})$, 
operators $h=h^{*} \in \mathcal{A}^{\prime \prime}, d \in \mathcal{A}^{\prime \prime} \otimes \mathrm{h}$ and contractions $w \in \mathcal{A}^{\prime \prime} \otimes \mathcal{B}\left(\mathrm{k}_{0} ; \mathrm{h}\right)$ and $v \in \mathcal{A}^{\prime \prime} \otimes \mathrm{k}_{0}$ such that, in the notation of $(1.1)^{\prime}$,

$$
\begin{gathered}
\widehat{\theta}(a)=\left[\begin{array}{cc}
\mathcal{L}(a)+\frac{1}{2}\{t, a\} & \delta^{\dagger}(a) w+a c^{*} \\
w^{*} \delta(a)+c a & w^{*} \pi(a) w
\end{array}\right] \\
\pi(1) d=d \text { and } \pi(1) w=w \\
\operatorname{Ran} \pi(1)=\mathrm{H} \\
c=\left(1-w^{*} w\right)^{1 / 2} v(-t)^{1 / 2}
\end{gathered}
$$

$\operatorname{Ker} v \supset \operatorname{Ker} t$ and $\operatorname{Ran} v \subset \overline{\operatorname{Ran}}\left(1-w^{*} w\right)$

where $t=\tau(1) \leq 0, c=\chi(1), \delta=\delta_{d, \pi}, \mathcal{L}=\mathcal{L}_{d, \pi, h}$ and $\mathrm{H}=\overline{\mathrm{H}_{0}}$ with

$$
\mathrm{H}_{0}=\operatorname{Lin}\left\{\delta(a) u^{0}+\pi(a) w\left(u^{i}\right): a \in \mathcal{A},\left(u^{\gamma}\right) \in \mathfrak{h}_{00}\right\} .
$$

Moreover, if $\mathcal{A}$ is a von Neumann algebra then the representation $(\pi, \mathfrak{h} \otimes \mathbf{h})$ of $\mathcal{A}$ appearing in $\mathcal{S}$ is normal if and only if the process $k$ is normal.

Proof. By Lemma 2.1 and [LW1], Proposition 5.1 and Theorem 5.2, (ii) implies (i). Conversely if (i) holds then $\theta$ is bounded and satisfies $\theta(1) \leq 0$, so that $\nu$ is a completely positive contraction. Letting $\left(\mathbf{h}, \pi, h, d,\left\{w_{i}\right\}\right)$ be the quintuple of Theorem 3.1, define an operator $w: \mathfrak{h} \otimes \mathrm{k}_{0} \rightarrow \mathfrak{h} \otimes \mathrm{h}$ by $w\left(v^{i}\right)=w_{i} v^{i}$, initially on vectors whose components are eventually zero. Since $\pi(1) w_{j}=w_{j}$ for each $j$, and

$$
\begin{aligned}
\left\|w\left(v^{i}\right)\right\|^{2}=\left\langle w_{i} v^{i}, w_{j} v^{j}\right\rangle & =\left\langle v^{i}, w_{i}^{*} \pi(1) w_{j} v^{j}\right\rangle \\
& =\left\langle v^{i}, \nu_{j}^{i}(1) v^{j}\right\rangle=\left\langle\left(v^{i}\right), \nu(1)\left(v^{i}\right)\right\rangle \leq\left\|\left(v^{i}\right)\right\|^{2},
\end{aligned}
$$

it follows that $w$ is a contraction. Since

$$
\left[\begin{array}{cc}
t & c^{*} \\
c & w^{*} w-1
\end{array}\right] \leq 0
$$

the existence of $v \in \mathcal{A}^{\prime \prime} \otimes \mathrm{k}_{0}$ satisfying (3.5iv) and (3.5v) follows from Lemma 2.1. The remaining properties now follow easily from Theorem 3.1, noting that when $\mathcal{A}$ is a von Neumann algebra, $k$ is normal if and only if each $\theta_{\beta}^{\alpha}$ is normal by [LW1], Proposition 3.2.

Remarks. (i) The necessary conditions (3.5) for $\theta$ to weakly generate a CP contraction cocycle $k$ have been shown to be sufficient too $([\mathbf{L W} 3])$, since completely bounded mapping matrices are necessarily regular in the sense of $[\mathrm{Mey}]$ and $[\mathbf{L W} \mathbf{1}]$. This also implies that $\theta$ strongly generates $k$. Uniqueness of solutions ([LW1], Theorem 3.1) permits us to use the notation $k^{\theta}$ for the cocycle generated by $\theta$.

(ii) By [LW1] Proposition 5.1, $k$ is unital if and only if $c$ and $t$ are both zero and $w$ is isometric; this implies that $v$ is zero too. 
Since we aim to dilate CP contraction cocycles to *-homomorphic cocycles, we need to be able to recognise from the generator when the flow is already *-homomorphic. This is addressed in the next result.

Proposition 3.3. Let $\mathcal{M}$ be a von Neumann algebra acting on $\mathfrak{h}$, and let $k$ be the flow generated by a (completely) bounded mapping matrix $\theta: \mathcal{M} \rightarrow$ $\mathcal{M} \otimes \mathcal{B}\left(\widehat{\mathrm{k}_{0}}\right)$ which is expressible in the form (3.5i) for some normal representation $(\pi, \mathfrak{h} \otimes \mathrm{h})$ of $\mathcal{M}$ and operators $c \in \mathcal{M} \otimes \mathrm{k}_{0}, h=h^{*} \in \mathcal{M}, d \in \mathcal{M} \otimes \mathrm{h}$ and $w \in \mathcal{M} \otimes \mathcal{B}\left(\mathrm{k}_{0} ; \mathrm{h}\right)$ satisfying (3.5ii). Then $k$ is ${ }^{*}$-homomorphic if and only if:

(i) $w$ is a partial isometry,

(ii) $w c=0$,

(iii) $c^{*} c=-t$,

(iv) $w w^{*} \in \pi(\mathcal{M})^{\prime}$, and

(v) $w w^{*} \delta=\delta$.

In particular, if $w w^{*}=\pi(1)$ then $k$ is ${ }^{*}$-homomorphic if and only if $w c=0$ and $c^{*} c=-t$.

Note. The hypotheses here imply complete positivity of $k$ (by Theorem 3.1) but not contractivity. If however $k$ is assumed to be contractive - equivalently $\theta(1) \leq 0$ - then there is an element $v$ of $\mathcal{M} \otimes \mathrm{k}_{0}$ satisfying (3.5iv), and so (i) implies (ii).

Proof. The necessary and sufficient conditions for $k$ to be ${ }^{*}$-homomorphic ([LW1], Theorem 6.5) may be written

$$
\begin{array}{r}
w^{*} \pi\left(a^{*} a\right) w=w^{*} \pi(a)^{*} w w^{*} \pi(a) w \\
w^{*} \delta\left(a^{*} a\right)=w^{*} \delta\left(a^{*}\right) a+w^{*} \pi\left(a^{*}\right) w\left(w^{*} \delta(a)+c a\right) \\
\left(w^{*} \delta(a)+c a\right)^{*}\left(w^{*} \delta(a)+c a\right)=\partial \tau(a, a)-a^{*} t a \\
=\delta(a)^{*} \delta(a)-a^{*} t a
\end{array}
$$

where (1.5) is used in the last line. Suppose (3.7)-(3.9) hold. Then, putting $a=1$ and using the fact that $\pi(1) w=w$, reveals that $\left(w^{*} w\right)^{2}=w^{*} w$, so that $w$ is a partial isometry. Now $\delta(1)=0$, so (3.8) and (3.9) with $a=1$ give $w c=0$ and $c^{*} c=-t$ respectively, which in turn implies that $\delta(a)^{*}\left(1-w w^{*}\right) \delta(a)=0$, and so $\left(1-w w^{*}\right) \delta(a)=0$. Also (3.7) implies that $w^{*} \pi(a)^{*}\left(1-w w^{*}\right) \pi(a) w=0$, so $\left(1-w w^{*}\right) \pi(a) w=0$, which implies that $\pi(a) w w^{*}=w w^{*} \pi(a) w w^{*}$, and taking adjoints shows that $w w^{*} \pi(a)=$ $\pi(a) w w^{*}$. Thus (i)-(iv) hold.

Conversely if (i)-(iv) hold then (3.7)-(3.9) are easily verified.

Remark. Invoking [GS] Theorem 3.3.6, instead of [LW1] Theorem 6.5, the proposition remains true when the noise dimension space is no longer separable. 


\section{4. *-Homomorphic dilation.}

In this section we give the main result of the paper, namely necessary and sufficient conditions for a Markovian cocycle to have a normal Markovregular *-homomorphic dilation. We then consider various special cases.

Theorem 4.1. Every normal Markov-regular completely positive contraction cocycle on a von Neumann algebra, with separable noise dimension space, admits a normal ${ }^{*}$-homomorphic stochastic dilation.

Proof. Let $k$ be a normal Markov-regular CP contraction cocycle on the von Neumann algebra $\mathcal{M}$ with separable noise dimension space $\mathrm{k}_{0}$. By Theorem 3.2 its stochastic generator $\theta$ satisfies

$$
\widehat{\theta}(a)=\left[\begin{array}{cc}
\tau(a) & \delta^{\dagger}(a) w+a c^{*} \\
w^{*} \delta(a)+c a & w^{*} \pi(a) w
\end{array}\right]=m \iota(a)+\iota(a) m^{*}+s^{*} \Psi(a) s
$$

where

$$
m=\left[\begin{array}{cc}
\frac{1}{2} t & 0 \\
c & 0
\end{array}\right] \in \mathcal{M} \otimes \mathcal{B}\left(\widehat{\mathrm{k}_{0}}\right), \quad s=\left[\begin{array}{cc}
1 & 0 \\
0 & w
\end{array}\right] \in \mathcal{M} \otimes \mathcal{B}\left(\widehat{\mathrm{k}_{0}} ; \widehat{\mathrm{h}}\right),
$$

$\Psi: \mathcal{M} \rightarrow \mathcal{M} \otimes \mathcal{B}(\widehat{\mathrm{h}})$ is the (transformed) mapping matrix

$$
\Psi=\left[\begin{array}{cc}
\mathcal{L} & \delta^{\dagger} \\
\delta & \pi
\end{array}\right]
$$

and (h, $\pi, h, d, w, v)$ is the sextuple $\mathcal{S}$ from the theorem satisfying (3.5). Set $\mathrm{k}=\mathrm{k}_{0} \oplus \mathrm{k}_{1} \oplus \mathrm{k}_{2}$ and let $\phi: \mathcal{M} \rightarrow \mathcal{M} \otimes \mathcal{B}(\widehat{\mathrm{k}})$ be the mapping matrix defined by

$$
\widehat{\phi}(a)=\left[\begin{array}{cc}
\tau(a) & \delta^{\dagger}(a) W+a C^{*} \\
W^{*} \delta(a)+C a & W^{*} \pi(a) W
\end{array}\right]=M \iota(a)+\iota(a) M^{*}+S^{*} \Psi(a) S
$$

where

$$
M=\left[\begin{array}{cc}
\frac{1}{2} t & 0 \\
C & 0
\end{array}\right] \in \mathcal{M} \otimes \mathcal{B}(\widehat{\mathrm{k}}) \text { and } S=\left[\begin{array}{cc}
1 & 0 \\
0 & W
\end{array}\right] \in \mathcal{M} \otimes \mathcal{B}(\widehat{\mathrm{k}} ; \widehat{\mathrm{h}}),
$$

with $W=\left[\begin{array}{lll}w & r & 0\end{array}\right]$ and $C=\left[\begin{array}{lll}c & g & e\end{array}\right]^{\top}$, and where the Hilbert spaces $\mathrm{k}_{1}$ and $\mathrm{k}_{2}$ and operators $r \in \mathcal{M} \otimes \mathcal{B}\left(\mathrm{k}_{1} ; \mathrm{h}\right), g \in \mathcal{M} \otimes \mathrm{k}_{1}$ and $e \in \mathcal{M} \otimes \mathrm{k}_{2}$ are to be determined.

Since $\phi$ is unchanged if $W$ is replaced by $\pi(1) W$, we may assume that $\pi(1) r=r$. By Lemma $1.2 k^{\phi}$ is a stochastic dilation of $k$, and by Proposition 3.3 and the remarks following it, $k^{\phi}$ is ${ }^{*}$-homomorphic if and only if

$$
q^{2}=q \in \pi(\mathcal{M})^{\prime}, q \delta=\delta, w c+r g=0 \text { and } c^{*} c+g^{*} g+e^{*} e=-t,
$$

where $q:=w w^{*}+r r^{*}$. The choice $\mathrm{k}_{1}=\mathrm{h}, \mathrm{k}_{2}=\mathbb{C}$ and

$$
r=\left(\pi(1)-w w^{*}\right)^{1 / 2}, \quad g=-w v(-t)^{1 / 2}, \quad e=\left(1-v^{*} v\right)^{1 / 2}(-t)^{1 / 2},
$$


satisfies these constraints since then $q=\pi(1)$, and by (3.5ii) and (3.5iv)

$$
\begin{aligned}
r g & =-\left(\pi(1)-w w^{*}\right)^{1 / 2} w v(-t)^{1 / 2} \\
& =-w\left(1-w^{*} w\right)^{1 / 2} v(-t)^{1 / 2}=-w c,
\end{aligned}
$$

and

$$
\begin{aligned}
t+g^{*} g+e^{*} e & =(-t)^{1 / 2}\left[-1+v^{*} w^{*} w v+\left(1-v^{*} v\right)\right](-t)^{1 / 2} \\
& =(-t)^{1 / 2} v^{*}\left(w^{*} w-1\right) v(-t)^{1 / 2}=-c^{*} c
\end{aligned}
$$

Remarks. The particular solution given above serves merely to establish the existence of stochastic dilations. More natural dilations arise in particular cases; moreover one may wish to preserve unitality under dilation. In the remarks below we simplify the constraints (4.2) by considering only dilations for which $q=\pi(1)$.

(i) If $w c=0$ then it is natural to choose $g=0$, simplifying the constraints to a question of finding $r \in \mathcal{M} \otimes \mathcal{B}\left(\mathrm{k}_{1} ; \mathrm{h}\right)$ and $e \in \mathcal{M} \otimes \mathrm{k}_{2}$ satisfying

$$
r r^{*}+w w^{*}=\pi(1) \text { and } c^{*} c+e^{*} e=-t .
$$

(ii) If $w$ is a partial isometry then $r$ is necessarily a partial isometry and (using (3.5iv)) $w c=0$ so (i) applies.

(iii) If $w w^{*}=\pi(1)$ then (ii) applies, and since $r$ is necessarily zero we may take $\mathrm{k}_{1}=\{0\}$. Moreover, by Proposition 3.3, $k$ is already ${ }^{*_{-}}$ homomorphic unless $c^{*} c \neq-t$, in which case we may take

$$
\mathrm{k}_{2}=\mathbb{C}, \quad C=\left[\begin{array}{c}
c \\
\left(-t-c^{*} c\right)^{1 / 2}
\end{array}\right] \text { and } W=\left[\begin{array}{ll}
w & 0
\end{array}\right] .
$$

(iv) If $k$ has a conservative Markov semigroup then $t=0$, and so $v$ and $c$ are zero. Also $e$ and $g$ are necessarily zero and we may take $\mathrm{k}_{2}=\{0\}$. By (iii) $k$ is already ${ }^{*}$-homomorphic unless $w w^{*} \neq \pi(1)$. In this case

$$
\widehat{\phi}(a)=S^{*} \Psi(a) S \text { for } S=\left[\begin{array}{ccc}
1 & 0 & 0 \\
0 & w & r
\end{array}\right],
$$

with $\Psi$ given by (4.1) and $r \neq 0$ satisfying $(4.2)^{\prime}$.

(v) If $k$ is unital then $\theta(1)=0$ (by [LW1], Proposition 5.1), (iv) applies and also $w$ is necessarily isometric so (ii) applies too. Thus we are left with a choice of Hilbert space $\mathrm{k}_{1}$ and partial isometry $r$ in $\mathcal{M} \otimes \mathcal{B}\left(\mathrm{k}_{1} ; \mathrm{h}\right)$ whose final space is $\operatorname{Ran}\left(\pi(1)-w w^{*}\right)$. The resulting dilation will be unital if and only if $r$ is isometric. In particular, the above scheme can yield a unital *-homomorphic dilation with the choice $\mathrm{k}_{1}=\mathrm{h}$ if and only if the projection $\left(\pi(1)-w w^{*}\right)$ is Murray-von Neumann equivalent to the identity, relative to the von Neumann algebra $\mathcal{M} \otimes \mathcal{B}(\mathrm{h})$. 
(vi) If $\mathrm{k}_{0}=\{0\}$ then there is no noise and so $k$ is a semigroup, $\mathcal{P}$ say. Thus $c, v$ and $w$ are all zero, in particular (ii) applies. If $\mathcal{P}$ is unital then (iv) and (v) apply too, and in this case $S=\operatorname{diag}[1, r]$. The choice $\mathrm{k}_{1}=\mathrm{h}$ and $r=\pi(1)$, so that $\widehat{\phi}=\Psi$, gives the dilation obtained in [GS]. If $\mathcal{P}$ is nonunital then, choosing $\mathrm{k}_{2}=\mathbb{C}$ and $\mathrm{k}_{1}=\mathrm{h}$ yields the generator

$$
\phi=\left[\begin{array}{ccc}
\tau & \delta^{\dagger} & \lambda^{\dagger} \\
\delta & \pi-\iota & 0 \\
\lambda & 0 & -\mathrm{id}
\end{array}\right],
$$

in which $\lambda$ denotes left multiplication by $e=(-t)^{1 / 2}$.

(vii) If $\mathrm{k}_{0}=\{0\}$ and $\mathcal{M}=\mathcal{B}(\mathfrak{h})$ where $\mathfrak{h}$ is infinite dimensional but separable, then (vi) applies and we may choose $\mathrm{k}_{1}$ to be (at most) one dimensional. To see this let

$$
\pi(a)=V^{*}\left(a \otimes 1_{\mathrm{h}^{\prime}}\right) V \text { with } V V^{*} \in 1_{\mathfrak{h}} \otimes \mathcal{B}\left(\mathrm{h}^{\prime}\right)
$$

be the normal decomposition of the representation $\pi$. Then, since $\mathrm{h}^{\prime}$ may be assumed to be separable, there is a partial isometry $U \in$ $\mathcal{B}\left(\mathfrak{h} ; \mathfrak{h} \otimes \mathrm{h}^{\prime}\right)$ with final space Ran $V V^{*}$. Putting $r=V^{*} U \in \mathcal{B}(\mathfrak{h} ; \mathfrak{h} \otimes \mathbf{h})$ yields the transformed dilation generator

$$
\widehat{\phi}=\left[\begin{array}{ccc}
\tau & \gamma^{\dagger} & \lambda^{\dagger} \\
\gamma & \nu & 0 \\
\lambda & 0 & 0
\end{array}\right],
$$

where $\nu(a)=U^{*}\left(a \otimes 1_{\mathrm{h}^{\prime}}\right) U$ and $\gamma=\delta_{D, \nu}$ for $D=U^{*} V d$. If $\partial \tau=0$ then $\delta=0$ by (1.5), and it follows from Theorem 1.3 that we may take $\mathrm{k}_{1}=\{0\}$ in (4.3) which (unless $\mathcal{P}$ is already ${ }^{*}$-homomorphic) gives the generator

$$
\phi=\left[\begin{array}{cc}
\tau & \lambda^{\dagger} \\
\lambda & -\mathrm{id}
\end{array}\right] .
$$

If $\partial \tau \neq 0$ then it follows that $\pi$ is nonzero and we may choose $U$ to be isometric, making $\nu$ unital. When $\mathcal{P}$ is unital, so that $\mathrm{k}_{2}$ may be chosen to be $\{0\}$, the resulting dilation is that obtained in $[\mathbf{H u S}]$.

Corollary 4.2 ([GS]). Every norm-continuous normal completely positive contraction semigroup on a von Neumann algebra has an Evans-Hudson dilation.

Corollary 4.3 ([HuS $])$. Let $\mathfrak{h}$ be infinite dimensional and separable. Every norm-continuous normal completely positive unital semigroup on $\mathcal{B}(\mathfrak{h})$ has a unital Evans-Hudson dilation with one-dimensional quantum noise. 


\section{Number/exchange-free dilation.}

In this section we seek necessary and sufficient conditions for a $\mathrm{CP}$ contraction cocycle to have a Markov-regular *-homomorphic stochastic dilation involving no number/exchange processes. Creation/annihilation-free dilations are also considered. Some interesting connections with locality, for quantum dynamical semigroups, emerge.

Proposition 5.1. Let $k$ be a Markov-regular completely positive contraction cocycle on a von Neumann algebra $\mathcal{M}$, with separable noise dimension space $\mathrm{k}_{0}$, and let $\theta=\left[\begin{array}{cc}\tau & \chi^{\dagger} \\ \chi & \nu-\iota_{0}\end{array}\right]$ be its stochastic generator and $\mathcal{P}$ its Markov semigroup.

(a) Suppose that $\nu=\iota_{0}$. Then the following are equivalent:

(i) $k$ has a number/exchange-free ${ }^{*}$-homomorphic dilation.

(ii) $k$ is unital, $\chi$ is an $\iota_{0}$-derivation and $\tau$ satisfies

$$
\partial \tau(a, a)-\chi(a)^{*} \chi(a)=\gamma(a)^{*} \gamma(a)
$$

where $\gamma: \mathcal{M} \rightarrow \mathcal{M} \otimes \mathrm{k}_{1}$ is an $\iota_{1}$-derivation for some Hilbert space $\mathrm{k}_{1}$.

(b) Suppose that $\chi=0$ and $k$ is normal. Then the following are equivalent:

(i) $k$ has a creation/annihilation-free ${ }^{*}$-homomorphic dilation.

(ii) $\mathcal{P}$ is ${ }^{*}$-homomorphic.

Proof. (a) Suppose that $k$ has a number/exchange-free *-homomorphic dilation. Then, by [LW1] Proposition 6.3 and Lemma 6.4, the generator of this dilation has the form

$$
a \mapsto\left[\begin{array}{cc}
l^{*}\left(a \otimes 1_{\mathrm{k}}\right) l-\frac{1}{2}\left\{l^{*} l, a\right\}+i[h, a] & a l^{*}-l^{*}\left(a \otimes 1_{\mathrm{k}}\right) \\
l a-\left(a \otimes 1_{\mathrm{k}}\right) l & 0
\end{array}\right]
$$

where $l \in \mathcal{M} \otimes \mathrm{k}, h=h^{*} \in \mathcal{M}$ and $\mathrm{k} \supset \mathrm{k}_{0}$. It follows that $\chi(a)=$ $m a-\left(a \otimes 1_{\mathrm{k}_{0}}\right) m$, where $m=p_{0} l \in \mathcal{M} \otimes \mathrm{k}_{0}, p_{0}$ being the orthogonal projection $\mathfrak{h} \otimes \mathfrak{k} \rightarrow \mathfrak{h} \otimes \mathrm{k}_{0}$, and so $\theta$ satisfies (ii).

Conversely, if $\theta$ has satisfies (ii) then by Theorem 1.3, $\chi(a)=m a-(a \otimes$ $\left.1_{\mathrm{k}_{0}}\right) m$ and $\gamma(a)=n a-\left(a \otimes 1_{\mathrm{k}_{1}}\right) n$ for some $m \in \mathcal{M} \otimes \mathrm{k}_{0}$ and $n \in \mathcal{M} \otimes \mathrm{k}_{1}$, and $\tau=\mathcal{L}_{l, \iota, h}$ where $\iota(a)=a \otimes 1_{\mathbf{k}_{0} \oplus \mathbf{k}_{1}}, l=[m n]^{\top}$ and $h=h^{*} \in \mathcal{M}$, so that $k$ has the number/exchange-free dilation with generator (5.2).

(b) First note that (ii) is equivalent to $\tau$ being a derivation. Since the creation component $\widetilde{\gamma}$ of a ${ }^{*}$-homomorphic dilation must satisfy $\tau\left(a^{*} a\right)$ $a^{*} \tau(a)-\tau(a)^{*} a=\widetilde{\gamma}(a)^{*} \widetilde{\gamma}(a)$ ([LW1], Section 6), (i) implies (ii).

Conversely, if (ii) holds then $\mathcal{P}$ is conservative and, since $k$ is normal, Remark (iv) above applies, with $\delta=0$, so $k$ has a creation/annihilation-free *-homomorphic dilation. 
Remark. From the structure of Markov-regular *-homomorphic generators without number/exchange coefficients (5.2) it is clear that on an abelian von Neumann algebra any nontrivial CP contraction cocycle requires number/exchange processes in its dilation. This is not so when the Markov semigroup is no longer assumed to be norm continuous $([\mathbf{G}])$.

Proposition 5.2. Let $k$ and $\theta$ be as in Proposition 5.1, and assume further that $\nu=\iota_{0}$ and $\chi$ is an $\iota_{0}$-derivation. If $k$ has a number/exchange-free ${ }^{*}$ homomorphic dilation then $k$ is unital and $\tau$ satisfies

$$
\partial \tau(a z, a z)=z^{*} \partial \tau(a, a) z \quad \forall a \in \mathcal{M}, z \in \mathcal{Z},
$$

where $\mathcal{Z}$ denotes the centre of $\mathcal{M}$. The converse holds when $\mathcal{M}^{\prime}$ is abelian and $k$ is normal.

Proof. The necessity of (5.3) is clear from the form $\tau$ must take according to (5.2). Conversely, suppose that $\mathcal{M}^{\prime}$ is abelian (and so equals $\mathcal{Z}$ ), $k$ is unital and normal and (5.3) holds, and let

$$
\theta(a)=\left[\begin{array}{cc}
\tau(a) & \chi^{\dagger}(a) \\
\chi(a) & \nu(a)-\iota_{0}(a)
\end{array}\right]=\left[\begin{array}{cc}
\mathcal{L}(a) & \delta^{\dagger}(a) w \\
w^{*} \delta(a) & 0
\end{array}\right]
$$

be a representation of $\theta$ according to Theorem 3.2. Thus

$$
w^{*} \pi(a) w=a \otimes 1_{\mathrm{k}_{0}} \text { and } \partial \mathcal{L}(a, a)=\delta(a)^{*} \delta(a),
$$

with $\pi$ normal. Also (5.3) with $a=1$, and (5.4), imply that $\delta(z)=0$ for $z \in \mathcal{Z}=\mathcal{M}^{\prime}$, so that

$$
\delta(a) a^{\prime}=\pi\left(a^{\prime}\right) \delta(a) \quad \forall a \in \mathcal{M}, a^{\prime} \in \mathcal{M}^{\prime} .
$$

Now (5.4) also implies that $w$ is isometric and, letting $p$ be the orthogonal projection $w w^{*} \in \mathcal{M} \otimes \mathcal{B}(\mathrm{h})$,

$$
\begin{aligned}
p \pi(a)^{*} p^{\perp} \pi(a) p & =p \pi\left(a^{*} a\right) p-p \pi\left(a^{*}\right) p \pi(a) p \\
& =w\left(a^{*} a \otimes 1_{\mathrm{k}_{0}}\right) w^{*}-w\left(a^{*} \otimes 1_{\mathrm{k}_{0}}\right)\left(a \otimes 1_{\mathrm{k}_{0}}\right) w^{*}=0,
\end{aligned}
$$

so $p$ commutes with $\pi(\mathcal{M})$ and thus $\pi_{1}(a)=p^{\perp} \pi(a) p^{\perp}$ defines a normal representation $\pi_{1}$ of $\mathcal{M}$. Let

$$
\pi_{1}(a)=V^{*}\left(a \otimes 1_{\mathrm{h}_{1}}\right) V \text { with } V V^{*} \in \mathcal{M}^{\prime} \otimes \mathcal{B}\left(\mathrm{h}_{1}\right),
$$

be its normal decomposition, and define a map $\gamma: \mathcal{M} \rightarrow \mathcal{B}\left(\mathfrak{h} ; \mathfrak{h} \otimes \mathrm{h}_{1}\right)$ by $\gamma=V \delta$. Note that $V$ satisfies

$$
\left(a \otimes 1_{\mathrm{h}_{1}}\right) V=V \pi(a) .
$$

By (5.5), (5.6) and (5.4), $\gamma$ satisfies

$$
\begin{gathered}
\gamma(a) a^{\prime}=V \pi\left(a^{\prime}\right) \delta(a)=\left(a^{\prime} \otimes 1_{\mathrm{h}_{1}}\right) \gamma(a) ; \\
\gamma(a b)-\gamma(a) b-\left(a \otimes 1_{\mathrm{h}_{1}}\right) \gamma(b)=V \pi(a) \delta(b)-\left(a \otimes 1_{\mathrm{h}_{1}}\right) V \delta(b)=0 ; \\
\gamma(a)^{*} \gamma(a)+\chi(a)^{*} \chi(a)=\delta(a)^{*}\left[V^{*} V+w w^{*}\right] \delta(a)=\partial \tau(a, a) ;
\end{gathered}
$$


for $a, b \in \mathcal{M}$ and $a^{\prime} \in \mathcal{M}^{\prime}$. Thus $\gamma$ is an $\iota_{1}$-derivation satisfying $\gamma(\mathcal{M}) \subset$ $\mathcal{M} \otimes \mathrm{h}_{1}$ and (5.1). The result follows.

Corollary 5.3. A number/exchange-free Markov-regular normal CP unital cocycle on $\mathcal{B}(\mathfrak{h})$ has a number/exchange-free *-homomorphic dilation if and only if the creation component of its generator is an $\iota_{0}$-derivation.

Remark. Two consequences of the existence of a number/exchange-free *homomorphic dilation for a Markov-regular CP contraction semigroup $\mathcal{P}$, with generator $\tau$, are

$$
\begin{gathered}
\partial \tau(a, z a)=\partial \tau(a, z) a+z \partial \tau(a, a) \\
\delta(a) z=0 \text { whenever } a z=0
\end{gathered}
$$

where $z \in \mathcal{Z}$ and $\delta$ is an $\iota_{1}$-derivation satisfying $\partial \tau(a, b)=\delta(a)^{*} \delta(b)$. When $\mathcal{M}$ is abelian (in which case $\mathcal{Z}=\mathcal{M}$ ) (5.7a) implies that

$\partial \tau$ is a derivation in its second argument.

In his study of locality for quantum dynamical semigroups ([Sa2]) Sauvageot (citing $[\mathbf{L e J}]$, Section 1.5) notes that, in the commutative $C^{*}$-case, $(5.7 \mathrm{a})^{\prime}$ is equivalent to the locality of $\mathcal{P}$; that is

$$
\lim _{t \searrow 0} t^{-1} \mathcal{P}_{t} f(x)=0 \text { whenever } f \text { vanishes in a neighbourhood of } x .
$$

This suggests an interesting link between the analytic property of locality and the quantum probabilistic property of number/exchange-free *-homomorphic dilatability, for noncommutative Markov semigroups. Note also that, in the commutative case, $(5.7 \mathrm{~b})$ is locality for $\delta$.

\section{Unitary dilation.}

In this section we consider contraction cocycles on a Hilbert space, and their stochastic dilation to unitary cocycles. We also apply this to construct an alternative form of dilation for unital CP cocycles on a von Neumann algebra.

A (left) Markovian contraction cocycle on the Hilbert space $\mathfrak{h}$ is a contraction process $\left(X_{t}\right)_{t \geq 0}$ on $\mathfrak{h}$ adapted to the Fock filtration and satisfying

$$
X_{s+t}=X_{s} \sigma_{s}\left(X_{t}\right),
$$

in which the right shift $\sigma_{s}$ acts on $\mathcal{B}\left(\mathfrak{h} \otimes \Gamma_{\mathfrak{k}}\right)$ by

$$
\sigma_{s}(T)=1_{s} \otimes S_{s} T S_{s}^{*},
$$

where $S_{s}$ is the right shift $\mathfrak{h} \otimes \Gamma_{\mathfrak{k}} \rightarrow \mathfrak{h} \otimes \Gamma_{[s, \infty[, \mathrm{k}}, 1_{s}$ is the identity operator on $\Gamma_{[0, s[, \mathrm{k}}$, and some natural identifications of tensor products are invoked. The Markov semigroup of $X$ is defined by $P_{t}=\mathbb{E}\left[X_{t}\right]=E^{*} X_{t} E(t \geq 0)$. The cocycle $X$ is called Markov-regular if $P$ is norm continuous $\mathbb{R}_{+} \rightarrow \mathcal{B}(\mathfrak{h})$. The counterpart to Theorem 1.1 is: 
Theorem 6.1 ([LW2]). Let $X$ be a contraction process on the Hilbert space $\mathfrak{h}$, with separable noise dimension space $\mathrm{k}_{0}$. Then the following are equivalent:

(i) $X$ is a Markov-regular cocycle.

(ii) $X$ weakly satisfies a $Q S$ differential equation of the form

$$
d X_{t}=X_{t} F_{\beta}^{\alpha} d \Lambda_{\alpha}^{\beta}(t), \quad X_{0}=1,
$$

for an operator matrix $F \in \mathrm{M}_{D}(\mathcal{B}(\mathfrak{h}))$.

In this case $X$ satisfies the equation strongly, and $F$ defines a bounded operator on $\mathfrak{h} \otimes \widehat{\mathrm{k}_{0}}$. Moreover, for a von Neumann algebra $\mathcal{M}$ acting on $\mathfrak{h}$, $X_{t} \in \mathcal{M} \otimes \mathcal{B}_{\mathrm{k}_{0}}$ for all $t \geq 0$ if and only if $F \in \mathcal{M} \otimes \mathcal{B}\left(\widehat{\mathrm{k}_{0}}\right)$.

Remark. A (unique) strong solution to (6.1) exists whenever $\left[F_{\beta}^{\alpha}\right]$ defines a bounded operator $F$ on $\mathfrak{h} \otimes \widehat{\mathrm{k}}([\mathbf{L W} \mathbf{1}]$, Theorem 7.1$)$, although the solution may be unbounded. Following (1.1) such $F$ will be written in block matrix form as $\left[\begin{array}{cc}A & B \\ C & D\end{array}\right]$ with $A \in \mathcal{B}(\mathfrak{h}), B, C^{*} \in \mathcal{B}(\mathfrak{h} \otimes \mathbf{k} ; \mathfrak{h})$ and $D \in \mathcal{B}(\mathfrak{h} \otimes \mathbf{k})$.

The case where $X$ is unitary was established in $[\mathbf{H u L}]$ and $[\mathbf{M o 1}]$. Weakly differentiable Markovian contraction cocycles were shown to satisfy a QS differential equation in $[\mathbf{A J L}]$ and $[\mathbf{F a g}]$. An interesting characterisation of strongly continuous unitary Markovian cocycles was obtained in [Jou], which also contains an example of such a cocycle which fails to satisfy a QS differential equation.

Propositions 7.5 and 7.6 of [LW1] may be stated as follows, using the representation of nonnegative operator matrices given in Lemma 2.1 once more:

Theorem 6.2. Let $X$ be a process on the Hilbert space $\mathfrak{h}$ with separable noise dimension space $\mathrm{k}_{0}$, weakly satisfying a QS differential equation of the form (6.1). Then the following equivalences hold:

(ai) $X$ is a contraction process.

(aii) $F$ is bounded with block matrix form

$$
F=\left[\begin{array}{cc}
i H-\frac{1}{2}\left(M^{*} M+B^{2}\right) & B V S-M^{*} W \\
M & W-1
\end{array}\right]
$$

where $H=H^{*}, B \geq 0,\|V\|,\|W\| \leq 1$ and $S=\left(1-W^{*} W\right)^{1 / 2}$.

(aiii) $F$ is bounded with block matrix form

$$
F=\left[\begin{array}{cc}
i H-\frac{1}{2}\left(L L^{*}+C^{2}\right) & -L \\
W L^{*}-R V^{\prime} C & W-1
\end{array}\right]
$$

where $H=H^{*}, C \geq 0,\left\|V^{\prime}\right\|,\|W\| \leq 1$ and $R=\left(1-W W^{*}\right)^{1 / 2}$.

(bi) $X$ is isometric.

(bii) In (aii) $W$ is isometric and $B=0$. 
(ci) $X$ is coisometric.

(cii) In (aiii) $W$ is coisometric and $C=0$.

(di) $X$ is unitary

(dii) In (aii) $W$ is unitary and $B=0$.

(diii) In (aiii) $W$ is unitary and $C=0$.

The representation (aii) is unique provided that $V$ satisfies $\operatorname{Ker} V \supset \operatorname{Ker} S$ and $\operatorname{Ran} V \subset \overline{\operatorname{Ran}} B$, which may be easily arranged. Uniqueness of the representation (aiii) may be similarly arranged.

Remark. Thus associated with a Markov-regular operator contraction cocycle $X$ is a unique octet $\left(H, W,(M, B, V),\left(L, C, V^{\prime}\right)\right)$, and conversely, $X$ is determined by either one of the two parameterisations $(H, W, M, B, V)$ or $\left(H, W, L, C, V^{\prime}\right)$.

Theorem 6.3. Every Markov-regular contraction cocycle on a Hilbert space, with separable noise dimension space, admits a unitary stochastic dilation.

Proof. Let $X$ be a Markov-regular contraction cocycle on $\mathfrak{h}$, with separable noise dimension space $\mathrm{k}_{0}$. By Theorems 6.1 and $6.2, X$ has a stochastic generator of the form

$$
\left[\begin{array}{cc}
K & -Q \\
N & W-1
\end{array}\right]
$$

where $K=i H-\frac{1}{2}\left(N^{*} N+B^{2}\right), Q=N^{*} W-B V S, H=H^{*}, B \geq 0$, $\|W\|,\|V\| \leq 1$ and $S=\left(1-W^{*} W\right)^{1 / 2}$.

Set $\mathrm{k}=\mathrm{k}_{0} \oplus \mathrm{k}_{1} \oplus \mathrm{k}_{2}$, where $\mathrm{k}_{1}$ and $\mathrm{k}_{2}$ are to be determined, and let $G \in \mathcal{B}(\mathfrak{h} \otimes \widehat{k})$ with block matrix form

$$
\left[\begin{array}{cc}
K & -L \\
M & U-1
\end{array}\right]
$$

in which

$$
U^{*} U=U U^{*}=1, M^{*} M=N^{*} N+B^{2} \text { and } L=M^{*} U .
$$

Then $G$ generates a unitary cocycle by Theorem 6.2 , and by the operator process analogue of Lemma 1.2 this cocycle is a stochastic dilation of $X$ if and only if

$$
M^{0}=N, L_{0}=Q \text { and } U_{0}^{0}=W .
$$

Solutions of the constraints (6.2) are obtained by taking $\mathrm{k}_{1}=\mathrm{k}_{0}, \mathrm{k}_{2}=\mathbb{C}$,

$$
M=\left[\begin{array}{c}
N \\
-V^{*} B \\
M_{2}
\end{array}\right], L=\left[\begin{array}{lll}
Q & L_{1} & M_{2}^{*}
\end{array}\right] \text {, and } U=\left[\begin{array}{ccc}
W & R & 0 \\
S & -W^{*} & 0 \\
0 & 0 & 1
\end{array}\right],
$$

where $R=\left(1-W W^{*}\right)^{1 / 2}, L_{1}=B V W^{*}+N^{*} R$, and $M_{2} \in \mathcal{B}(\mathfrak{h})$ is chosen so that $M_{2}^{*} M_{2}=B\left(1-V V^{*}\right) B$. 
Remarks. (i) If $X$ is affiliated to a von Neumann algebra $\mathcal{M}$, then it has a unitary dilation which is affiliated to $\mathcal{M}$ also.

(ii) If $B=0$, in particular if $X$ is isometric (but not unitary), then $M_{2}=0$ and we may take $\mathrm{k}_{1}=\mathrm{k}_{0}$ and $\mathrm{k}_{2}=\{0\}$, giving the dilation generator

$$
G=\left[\begin{array}{ccc}
i H-\frac{1}{2} N^{*} N & -N^{*} W & -N^{*} R \\
N & W-1 & R \\
0 & S & -W^{*}-1
\end{array}\right],
$$

with $R$ and $S$ as above.

(iii) If $W$ is unitary (but $B \neq 0$ ) then $V=0$ and the simplest solution is $\mathrm{k}_{1}=\{0\}, \mathrm{k}_{2}=\mathbb{C}$ and

$$
G=\left[\begin{array}{ccc}
i H-\frac{1}{2}\left(N^{*} N+B^{2}\right) & -N^{*} W & i B \\
N & W-1 & 0 \\
i B & 0 & 0
\end{array}\right] .
$$

(iv) To see what is being stochastically generalised here, let $k_{0}=\{0\}$ so that we have a contraction semigroup on $\mathfrak{h}$. Then (iii) applies, giving

$$
G=\left[\begin{array}{cc}
i H-\frac{1}{2} B^{2} & i B \\
i B & 0
\end{array}\right],
$$

and the dilation is effected by the solution $U$ of the stochastic differential equation

$$
d U_{t}=U_{t}\left[i B d Q_{t}+\left(i H-\frac{1}{2} B^{2}\right) d t\right]
$$

where $Q=\left(A_{t}+A_{t}^{\dagger}\right)_{t \geq 0}$ is a classical Brownian motion. When the operators $B$ and $H$ commute it is given explicitly by

$$
U_{t}=e^{i\left(B \otimes Q_{t}+t H \otimes I\right)} .
$$

(v) Using time-reversal techniques, or by taking adjoints, Markov-regular right contraction cocycles are equally seen to have unitary stochastic dilations.

We end this section with an alternative form of dilation for unital CP cocycles.

Theorem 6.4. Every normal Markov-regular completely positive unital cocycle $k$, on a von Neumann algebra, with separable noise dimension space $\mathrm{k}_{0}$ and initial space $\mathfrak{h}$, enjoys a factorisation of the following form:

$$
\widetilde{k}_{t}=\mathbb{E}_{1} \circ \operatorname{ad} U_{t} \circ \iota
$$

in which $\widetilde{k}_{t}(a)=k_{t}(a) \otimes 1$ on $\mathfrak{h} \otimes \Gamma_{\mathrm{k}_{1}}, \iota(a)=a \otimes 1$ on $\mathfrak{h} \otimes \Gamma_{\mathrm{k}_{1} \oplus \mathrm{k}_{1}}, \mathrm{k}_{1} \supset \mathrm{k}_{0}$ is separable and $U$ is a Markov-regular unitary cocycle. If $\mathrm{k}_{0}$ is infinite dimensional then we may take $\mathrm{k}_{1}=\mathrm{k}_{0}$, so that $\widetilde{k}=k$. 
Proof. Theorem 4.5 of [GLW] ensures the existence of a separable Hilbert space $\mathrm{k}_{1}$ containing $\mathrm{k}_{0}$, and a Markov-regular coisometry cocycle $X$ with noise dimension space $\mathrm{k}_{1}$ such that $k_{t}(a) \otimes 1=X_{t}(a \otimes 1) X_{t}^{*}$ on $\mathfrak{h} \otimes \Gamma_{\mathrm{k}_{1}}$. Let $k^{-}$denote the Markovian cocycle on $\mathcal{B}(\mathfrak{h})$ given by the same formula. The stochastic generators of $X$ and $k^{-}$have the respective forms

$$
\widehat{F}=\left[\begin{array}{cc}
i H-\frac{1}{2} M^{*} M & -M^{*} \\
W^{*} M & W^{*}
\end{array}\right], \quad \widehat{\theta}(a)=\left[\begin{array}{cc}
\tau(a) & \delta^{\dagger}(a) W \\
W^{*} \delta(a) & W^{*} \iota_{1}(a) W
\end{array}\right]
$$

where $H=H^{*}, W^{*} W=1, \iota_{1}(a)=a \otimes 1$ on $\mathfrak{h} \otimes \mathbf{k}_{1}, \delta=\delta_{M, \iota_{1}}$ and $\tau=\mathcal{L}_{M, \iota_{1}, H}$. If $X$ is unitary then (6.3) holds with $U_{t}=X_{t} \otimes 1$. If $X$ is nonunitary then, by Theorem $6.2, W^{*}$ is nonisometric and we let $U$ be the unitary stochastic dilation of $X$ whose generator is defined by

$$
\widehat{G}=\left[\begin{array}{ccc}
i H-\frac{1}{2} M^{*} M & -M^{*} & 0 \\
W^{*} M & W^{*} & 0 \\
R M & R & -W
\end{array}\right]
$$

where $R=\left(1-W W^{*}\right)$, and set $j_{t}^{-}=\operatorname{ad} U_{t}: a \mapsto U_{t}(a \otimes 1) U_{t}^{*}$. Then the stochastic generator of $j^{-}$is given by

$$
\phi(a)=\iota(a) G^{*}+G \iota(a)+G \Delta(a) G^{*},
$$

where $\Delta(a)=a \otimes \Delta$ and $\Delta$ is the orthogonal projection in $\mathcal{B}(\widehat{\mathrm{k}})$ with range $\mathrm{k}=\mathrm{k}_{1} \oplus \mathrm{k}_{1}$, so that

$$
\widehat{\phi}(a)=\left[\begin{array}{ccc}
\tau(a) & \delta^{\dagger}(a) W & \delta^{\dagger}(a) R \\
W^{*} \delta(a) & W^{*} \iota_{1}(a) W & W^{*} \iota_{1}(a) R \\
R \delta(a) & R \iota_{1}(a) W & R \iota_{1}(a) R+W \iota_{1}(a) W^{*}
\end{array}\right] .
$$

Invoking Lemma 1.2 once more, comparison with (6.4) shows that $j^{-}$is a stochastic dilation of $k^{-}$, and the result follows.

Remark. This need not be a stochastic dilation of $\widetilde{k}$ in our sense, since $U$ need not be affiliated to $\mathcal{M}$.

\section{Application to classical probability.}

Two areas of classical probability where ideas from the theory of CP-valued quantum processes might be applied are filtering theory and measure-valued diffusion. Indeed the Zakai equation (see e.g., $[\mathbf{K a K}]$ ), governing the conditional distribution of a signal process at time $t$ given the $\sigma$-algebra of an observed process up to time $t$, was a motivation for Belavkin to consider quantum filtering from the point of view of QS differential equations $([\mathrm{Be} 1])$. Below we give a rough outline of how one might view measurevalued diffusions as conditioned classical processes on the underlying state space.

Consider a compact Hausdorff space $X$. Let $\mathcal{P}$ denote the set of regular Borel probability measures on $X$, equipped with the topology of weak 
convergence, and let $\mathcal{A}$ denote the unital $C^{*}$-algebra of continuous complexvalued functions on $X$. Also let $L^{2}(\mathbb{P})$ be the $L^{2}$-space of the probability space $(\Omega, \mathfrak{F}, \mathbb{P})$ of a family $P^{1}, \ldots, P^{m}$ of independent Poisson processes with respective intensities $\lambda_{1}, \ldots, \lambda_{m}$. Finally let $\mu:=\left(\mu_{t}^{x}: \Omega \rightarrow \mathcal{P}\right)_{x \in X, t \geq 0}$ be a family of maps satisfying suitable measurability conditions, the condition that, for each $t \geq 0$ and $f \in \mathcal{A}$, the map $x \mapsto\left\langle f, \mu_{t}^{x}(\omega)\right\rangle:=\int f(y) \mu_{t}^{x}(\omega)(d y)$ is continuous for almost all $\omega \in \Omega$, and also the following stochastic differential equation:

$$
d\left\langle f, \mu_{t}^{x}\right\rangle=\left\langle\alpha_{0}(f), \mu_{t}^{x}\right\rangle d t+\sum_{i=1}^{m}\left\langle\alpha_{i}(f), \mu_{t}^{x}\right\rangle d P_{t}^{i}, \quad \mu_{0}^{x}=\delta_{x},
$$

where $\alpha_{0}, \ldots, \alpha_{m}$ are bounded linear maps on $\mathcal{A}$ such that $\alpha_{i}(\bar{f})=\overline{\alpha_{i}(f)}$ and $\alpha_{i}(1)=0$. Thus $\left(\mu_{t}^{x}\right)$ is a measure-valued diffusion.

In order to cast this in a QS form, first let $\mathcal{M}$ be the universal enveloping algebra of $\mathcal{A}$ and let $\mathfrak{h}$ be the Hilbert space on which it acts. Then each $\alpha_{i}$ extends to a bounded normal operator on $\mathcal{M}$ - which we denote by $\alpha_{i}^{* *}$.

Under the natural identification of $L^{2}(\mathbb{P})$ with $\Gamma\left(L^{2}\left(\mathbb{R}_{+} ; \mathbb{C}^{m}\right)\right)$, the operator of multiplication by $P_{t}^{i}$ corresponds to the following combination of fundamental quantum processes:

$$
N_{t}^{i}(t)+\sqrt{\lambda_{i}}\left(A_{i}(t)+A_{i}^{\dagger}(t)\right)+\lambda_{i} t
$$

([Mey], p. 74). Let $\bar{k}$ be the Markovian cocycle on $\mathcal{M}$ generated by $\theta$ where

$$
\theta_{0}^{0}=\alpha_{0}^{* *}+\sum_{i=1}^{m} \lambda_{i} \alpha_{i}^{* *}, \theta_{0}^{i}=\theta_{i}^{0}=\sqrt{\lambda_{i}} \alpha_{i}^{* *}, \text { and } \theta_{j}^{i}=\delta_{j}^{i} \alpha_{i}^{* *} .
$$

Then $\bar{k}$ is normal, CP and unital. Comparison with (7.1) and (7.2) shows that for $f \in \mathcal{A}$, viewed as a subalgebra of $\mathcal{M}, k_{t}(f)$ corresponds to the operator of multiplication by the function $F_{t}: \Omega \rightarrow \mathcal{A} \subset \mathcal{M}$ given by $F_{t}(\omega)=\left\langle f, \mu_{t}(\omega)\right\rangle$.

Suppose now that $\bar{k}$ has a unital ${ }^{*}$-homomorphic dilation $\bar{\jmath}$. Let $j_{t}$ be the restriction of $\bar{j}_{t}$ to $\mathcal{A}$ then the $C^{*}$-algebra generated by $\left\{j_{t}(f): t \geq\right.$ $0, f \in \mathcal{A}\}$ is abelian ([Par], Theorem 28.8). Letting $\Sigma$ be its spectrum, our process may be viewed as consisting of unital ${ }^{*}$-homomorphisms $j_{t}$ : $C(X) \rightarrow C(\Sigma)$. By Gelfand theory (specifically the Banach-Stone Theorem) there are continuous maps $\xi_{t}: \Sigma \rightarrow X$ such that $j_{t}(f)=f \circ \xi_{t}$. In this way the dilation of $k$ may be viewed as a lifting of the measure-valued diffusion $\mu$ to a "process" $\xi$ taking values in the underlying state space.

Acknowledgements. DG is grateful for financial support from the Indian National Board of Higher Mathematics (Department of Atomic Energy); JML and SJW are grateful to the Delhi Centre of the Indian Statistical Institute for hospitality; SJW's stay at ISI was financed by the Royal SocietyIndian National Science Academy exchange programme; KBS acknowledges 
support from the Jawaharlal Nehru Centre for Advanced Scientific Research, Bangalore. DG also acknowledges the support of Av Humboldt Stiftung (Germany).

\section{References}

[AAFL] L. Accardi, R. Alicki, A. Frigerio and Y.-G. Lu, An invitation to the weak coupling and low density limits, in 'Quantum Probability and Related Topics VI,' edited by L. Accardi, World Scientific, Singapore, 1991, 3-61, CMP 1149 817, Zbl 0927.60090.

[AJL] L. Accardi, J.-L. Journé and J.M. Lindsay, On multi-dimensional Markovian cocycles, in 'Quantum Probability and Applications IV,' edited by L. Accardi and W. von Waldenfels, Lecture Notes in Mathematics, 1396, Springer-Verlag, Heidelberg, 1989, 59-67, MR 92a:81086, Zbl 0674.60064.

[AcM] L. Accardi and A. Mohari, On the structure of classical and quantum flows, J. Funct. Anal., 135 (1996), 421-455, MR 96m:46115, Zbl 0837.60090.

[Be1] V.P. Belavkin, Quantum stochastic calculus and quantum nonlinear filtering, J. Multivariate Anal., 42 (1992), 171-201, MR 93m:81013, Zbl 0762.60059.

[Be2] V.P. Belavkin, Quantum stochastic positive evolutions: Characterization, construction, dilation, Comm. Math. Phys., 184 (1997), 533-566, MR 98k:81132, Zbl 0874.60073.

[Bha] B.V. Rajarama Bhat, An index theorem for quantum dynamical semigroups, Trans. Amer. Math. Soc., 348 (1996), 561-583, MR 96g:46059, Zbl 0842.46045.

[Bra] W.S. Bradshaw, Stochastic cocycles as a characterisation of quantum flows, Bull. Sci. Math. (2), 116 (1992), 1-34, MR 93c:46116, Zbl 0820.60096.

[ChE] E. Christensen and D.E. Evans, Cohomology of operator algebras and quantum dynamical semigroups, J. London Math. Soc., 20 (1979), 358-368, MR 81f:46082, Zbl 0448.46040.

[Dix] J. Dixmier, Von Neumann Algebras, North-Holland, Amsterdam, 1981, MR 83a:46004, Zbl 0473.46040.

[EvL] D.E. Evans and J.T. Lewis, Dilations of irreversible evolutions in algebraic quantum theory, Comm. Dublin Inst. Adv. Studies Ser. A, 24, 1977, MR 58 \#8915, Zbl 0365.46059.

[EH1] M.P. Evans and R.L. Hudson, Multidimensional quantum diffusions, in 'Quantum Probability and Applications III', edited by L. Accardi and W. von Waldenfels, Lecture Notes in Mathematics, 1303, Springer-Verlag, Heidelberg, 1988, 69-88, MR 91a:46065, Zbl 0648.46056.

[EH2] - Perturbations of quantum diffusions, J. London Math. Soc., 41 (1990), 373-384, MR 92a:46072, Zbl 0719.60083.

[Fag] F. Fagnola, Characterization of isometric and unitary weakly differentiable cocycles in Fock space, in 'Quantum Probability and Related Topics VIII', edited by L. Accardi, World Scientific, Singapore, 1993, 143-164, MR 95e:81122.

[FoF] C. Foias and A.E. Frazho, The commutant lifting approach to interpolation problems, Operator Theory: Advances and Applications, 44, Birkhäuser Verlag, Basel, 1990, MR 92k:47033, Zbl 0718.47010. 
[G] D. Goswami, Quantum Stochastic Dilation of Completely Positive Semigroups and Flows, Ph.D. Thesis, Indian Statistical Institute, Delhi, 1999.

[GLW] D. Goswami, J.M. Lindsay and S.J. Wills, A stochastic Stinespring theorem, Math. Ann., 319 (2001), 647-673, MR 2002c:46124, Zbl 0981.46052.

[GS] D. Goswami and K.B. Sinha, Hilbert modules and stochastic dilation of a quantum dynamical semigroup on a von Neumann algebra, Comm. Math. Phys., 205 (1999), 377-405, MR 2000k:81160, Zbl 0935.46055.

[HuL] R.L. Hudson and J.M. Lindsay, On characterizing quantum stochastic evolutions, Math. Proc. Cambridge Philos. Soc., 102 (1987), 363-369, MR 88m:81041, Zbl 0644.46046.

[HuP] R.L. Hudson and K.R. Parthasarathy, Quantum Itô's formula and stochastic evolutions, Comm. Math. Phys., 93 (1984), 301-323, MR 86e:46057, Zbl 0546.60058.

[HuS] R.L. Hudson and P. Shepperson, Stochastic dilation of quantum dynamical semigroups using one-dimensional quantum stochastic calculus, in 'Quantum Probability and Applications V', edited by L. Accardi and W. von Waldenfels, Lecture Notes in Mathematics, 1442, Springer-Verlag, Heidelberg, 1990, 216-218, MR 92a:81092, Zbl 0706.60067.

[Jou] J-L. Journé, Structure des cocycles markoviens sur l'espace de Fock, Probab. Theory Related Fields, 75 (1987), 291-316, MR 88g:46071, Zbl 0595.60066.

[KaK] G. Kallianpur and R.L. Karandikar, White Noise Theory of Prediction, Filtering and Smoothing, Gordon and Breach Science Publishers, New York, 1988, MR 90a:60086, Zbl 0724.93076.

[Küm] B. Kümmerer, Markov dilations on $W^{*}$-algebras, J. Funct. Anal., 63 (1985), 139177, MR 87b:46070, Zbl 0601.46062.

[LeJ] Y. Le Jan, Mesures associées à une forme de Dirichlet. Applications, Bull. Soc. Math. France, 106 (1978), 61-112, MR 81c:31014, Zbl 0393.31008.

[LiP] J.M. Lindsay and K.R. Parthasarathy, On the generators of quantum stochastic flows, J. Funct. Anal., 158 (1998), 521-549, MR 99k:81145, Zbl 0914.60033.

[LW1] J.M. Lindsay and S.J. Wills, Existence, positivity, and contractivity for quantum stochastic flows with infinite dimensional noise, Probab. Theory Related Fields, 116 (2000), 505-543, MR 2002a:81149.

[LW2] - Markovian cocycles on operator algebras, adapted to a Fock filtration, J. Funct. Anal., 178 (2000), 269-305, MR 2001m:81149, Zbl 0969.60066.

[LW3] Existence of Feller cocycles on a $C^{*}$-algebra, Bull. London Math. Soc., 33 (2001), 613-621, MR 2002e:81106.

[Mey] P-A. Meyer, Quantum Probability for Probabilists, 2nd Edition, Lecture Notes in Mathematics, 1538, Springer-Verlag, Heidelberg, 1993, MR 94k:81152, Zbl 0773.60098.

[Mo1] A. Mohari, Quantum stochastic differential equations with unbounded coefficients and dilations of Feller's minimal solution, Sankhyā Ser. A, 53 (1991), 255-287, MR 93m:81083, Zbl 0751.60062.

[Mo2] _ Quantum Stochastic Calculus with Infinite Degrees of Freedom, Ph.D. Thesis, Indian Statistical Institute, Delhi, 1992.

[MoS] A. Mohari and K.B. Sinha, Quantum stochastic flows with infinite degrees of freedom and countable state Markov processes, Sankhyā Ser. A, 52 (1990), 43-57, MR 93i:81112, Zbl 0719.60126. 
[Par] K.R. Parthasarathy, An Introduction to Quantum Stochastic Calculus, Birkhäuser, Basel, 1992, MR 93g:81062, Zbl 0751.60046.

[Sa1] J.-L. Sauvageot, Markov quantum semigroups admit covariant Markov $C^{*}$ dilations, Comm. Math. Phys., 106 (1986), 91-103, MR 88a:46077, Zbl 0606.60079.

[Sa2] - Tangent bimodule and locality for dissipative operators on $C^{*}$-algebras, in 'Quantum Probability and Applications IV,' edited by L. Accardi and W. von Waldenfels, Lecture Notes in Mathematics, 1396, Springer-Verlag, Berlin, 1989, 322-338, MR 90k:46145, Zbl 0682.46042.

[Vin] G.F. Vincent-Smith, Dilation of a dissipative quantum dynamical system to a quantum Markov process, Proc. London Math. Soc., 49 (1984), 58-72, MR 85i:46086, Zbl 0572.46055.

Received Received December 15, 1999 and revised January 10, 2001.

MATH-Stat Unit

Indian Statistical Institute (Kolkata Centre)

203 Barrackpore Trunk Road

KOLKATA 700108

INDIA

E-mail address: goswamid@isical.ac.in

School of Mathematical Sciences

UNIVERSITY OF NOTTINGHAM

University Park, Nottingham, NG7 2RD

$\mathrm{UK}$

E-mail address: jml@maths.nott.ac.uk

Indian Statistical Institute (Delhi Centre)

7 SJS SANSANWAL Marg

New Delhi - 110016

INDIA

E-mail address: kbs@isid.ac.in

Department of Mathematics

University College, Cork

IRELAND

E-mail address: s.wills@ucc.ie 